\title{
Conservative dynamics of binary systems to fourth post-Newtonian order in the EFT approach. II. Renormalized Lagrangian
}

\author{
Stefano Foffa, ${ }^{1}$ Rafael A. Porto, ${ }^{2,3}$ Ira Rothstein, ${ }^{4}$ and Riccardo Sturani ${ }^{5}$ \\ ${ }^{1}$ Département de Physique Théorique and Center for Astroparticle Physics, Université de Geneve, \\ 24 quai Ansermet, $\mathrm{CH}-1211$ Geneve 4, Switzerland \\ ${ }^{2}$ Deutsches Elektronen-Synchrotron DESY, Notkestraße 85, D-22603 Hamburg, Germany \\ ${ }^{3}$ The Abdus Salam International Center for Theoretical Physics, Strada Costiera, 11, Trieste 34151, Italy \\ ${ }^{4}$ Department of Physics and Astronomy Carnegie Mellon University, \\ Pittsburgh, Pennsylvania 15213, USA \\ ${ }^{5}$ International Institute of Physics (IIP), Universidade Federal do Rio Grande do Norte (UFRN) \\ CP 1613, 59078-970 Natal-RN Brazil
}

(Received 12 April 2019; published 24 July 2019)

\begin{abstract}
We complete the derivation of the conservative dynamics of binary systems to fourth Post-Newtonian (4PN) order in the effective field theory (EFT) approach. We present a self-contained (ambiguity-free) computation of the renormalized Lagrangian, entirely within the confines of the PN expansion. While we confirm the final results reported in the literature, we clarify several issues regarding intermediate infrared (IR) and ultraviolet (UV) divergences, as well as the renormalization procedure. First, we properly identify the IR and UV singularities using (only) dimensional regularization and the method of regions, which are the pillars of the EFT formalism. This requires a careful study of scaleless integrals in the potential region, as well as conservative contributions from radiation modes due to tail effects. As expected by consistency, the UV divergences in the near region (due to the point-particle limit) can be absorbed into two counterterms in the worldline effective theory. The counterterms can then be removed by field redefinitions, such that the renormalization scheme dependence has no physical effect to 4PN order. The remaining IR poles, which are spurious in nature, are unambiguously removed by implementing the zero-bin subtraction in the EFT approach. The procedure transforms the IR singularities into UV counterparts. As anticipated, the leftover UV poles explicitly cancel out against UV divergences in conservative terms from radiation reaction, uniquely determining the gravitational potential. Similar artificial IR/UV poles, which are intimately linked to the split into regions, are manifest at lower orders. Starting at 4PN order, both localand nonlocal-in-time contributions from the radiation region enter in the conservative dynamics. Neither additional regulators nor ambiguity parameters are introduced at any stage of the computations.
\end{abstract}

DOI: $10.1103 /$ PhysRevD.100.024048

\section{INTRODUCTION}

The detection of gravitational waves (GWs) by the LIGO/Virgo Collaboration [1] has initiated an unprecedented new epoch for explorations of the Universe. After the remarkable historical detections, GW science will soon turn into the study of the properties of the sources, addressing foundational questions in astrophysics, cosmology, and particle physics [2-4]. In particular, binary systems of comparable masses or extreme-mass ratios are posed to become the leading probe to test gravitational dynamics and the physics of compact objects-such as black holes and neutron stars-under unique conditions. The number of events observed up to now demonstrates the feasibility of the direct detection of GWs over a large range of sources [5]. We expect several events per year once current detectors are running at designed sensitivity, and many more with future observatories. Precise theoretical templates are thus a compulsory ingredient for data analysis and the reliable physical interpretation of the signals obtained with present and planned GW detectors. As a result, the two-body problem in gravity has become a very active area of research, relying on both numerical and analytical methodologies [2]. A large portion of the GW signal is emitted during the inspiral phase, which in principle can be understood analytically using the postNewtonian (PN) formalism, where traditional methods in general relativity have a long and rich history; see, e.g., Refs. [6,7]. More recently, the effective field theory (EFT) framework introduced in Ref. [8] has become a powerful new method to solve the two-body problem, successfully extending the knowledge of the binary's dynamics to high PN orders [9-13]. The purpose of this paper is to continue the path towards precision gravitational waveforms, by completing the derivation of the gravitational potential for 
nonspinning compact objects to fourth PN (4PN) order within the EFT approach, building upon the results reported in a companion paper [14] and elsewhere [15-20].

The Hamiltonian (and Lagrangian) for the conservative dynamics at 4PN order was first reported in Refs. [21-25] and Refs. [26,27] in the Arnowitt-Deser-Misner (ADM) [7] and "Fokker-action" [6] approaches, respectively. Yet, the introduction of "ambiguity parameters" due to the presence of infrared (IR) divergences, in addition to the ultraviolet (UV) ones, together with further claims for more ambiguities to address a discrepancy between the two first independent derivations [25-27] appeared to signal a breakdown of the split into regions at $4 \mathrm{PN}$ order. As a consequence, the ambiguities were resolved originally by relying on information outside of the PN framework $[22,23,27]$. Later on, the rederivation in Refs. [28,29] of the conservative contribution in radiation reaction due to the tail effect using dimensional regularization (dim. reg.), confirming the result obtained in Refs. [16,17] within the EFT approach, provided the last ingredient to fix the two ambiguities introduced in Refs. [26,27]. This completed the calculation within the Fokker-action formalism, without the need of extra matching conditions. The one ambiguity parameter in the ADM approach [21,23,24] has, thus far, only been obtained by incorporating results from gravitational self-force calculations [22].

Even though in practice the ambiguities introduced in Refs. [21,23-27] were determined and the complete result reported, the derivations in both the ADM [21,24] and Fokker-action [28,29] formalisms left room open for further improvement and clarifications, in particular, with regards to the handling of IR divergences (which led to the introduction of ambiguity parameters in the first place) and the apparent reliance on an extra regulator beyond dim. reg. ${ }^{1}$ Moreover, while the renormalization procedures presented elsewhere led to the correct result, ${ }^{2}$ a more systematic removal of IR/UV divergences will be needed when physical logarithms in the near zone first appear at higher PN orders (due to finite-size effects). We address all of these issues in the present paper, by providing an ambiguity-free and systematic derivation of the renormalized Lagrangian in dim. reg., all within the confines of the PN expansion, which can be naturally extended to all orders.

\footnotetext{
${ }^{1}$ A combined (" $\epsilon B$ ") dimensional and analytic regularization is used in the ADM formalism (see Appendix A in Ref. [24]) while, similarly, a combined (" $\epsilon \eta$ ") regularization is implemented in the Fokker-action approach [see, e.g., the paragraphs after Eq. (3.6) in Ref. [28] and after Eqs. (2.5)-(2.6) in Ref. [29] ].

${ }^{2}$ For instance, a (short-distance) worldline shift was implemented in Ref. [26] (see, e.g., their Appendix C). However, it includes both (long-distance) IR poles from the potential region and UV poles from tail terms combined, together with their associated length scales.
}

As discussed in Refs. $[17,19,20]$, the EFT formalism clearly illustrates the origin of the apparent ambiguities due to the split into regions, while already providing the unambiguous contributions from the tail effect to the effective Lagrangian. However, the computation of the local-in-time near-zone regularized Lagrangian in the EFT approach was (until now) pending, with intermediate results at orders $G, G^{2}$, and $G^{5}$ presented elsewhere [15,18] (see also Ref. [30]). ${ }^{3}$ In a companion paper [14], the remaining $G^{3}$ and $G^{4}$ contributions are reported, using dim. reg. to handle the divergences. We point the reader to Ref. [14] for a thorough derivation of the relevant Feynman diagrams which contribute to this order. While in the near region the singular terms in the limit $d \rightarrow 3$ were identified in Ref. [14], the distinction between IR and UV poles was not addressed. This is crucial for the proper renormalization of the effective theory. One of the goals of this paper is therefore to perform a careful analysis of divergent integrals in dim. reg., identifying the type of near- and far-zone singularities. As we shall see, scaleless (self-energy) integrals as well as conservative radiation-reaction terms play a key role.

After we isolate the coefficients of the IR and UV poles, we perform the systematic renormalization of the effective theory. By identifying the IR/UV singularities, the elimination of UV poles in the potential region can be performed without knowledge of contributions from radiation modes, as expected. These UV poles can be absorbed into counterterms in the point-particle action, which in turn can be removed by field redefinitions, as emphasized in Ref. [8]. Therefore, the renormalization scheme dependence has no physical effect to 4PN order and, for simplicity, we will choose a minimal-subtraction (MS) scheme. Once the UV poles are renormalized away, the remaining IR divergences - arising in the near zone due to an overlap (double counting) between regions of integration-are handled by the zero-bin subtraction [33] applied to the EFT approach [19,20]. The procedure unambiguously removes the IR poles, transforming them into UV counterparts. The leftover poles cancel out against divergences arising in conservative radiation-reaction terms, uniquely fixing the gravitational potential at 4PN order, as emphasized in Refs. $[17,19,20]{ }^{4}$

As we shall see, the link between IR/UV divergences appears already at lower orders, as it is required by consistency of the split into regions, albeit with contributions that are proportional to conserved currents or vanish on shell. The situation changes at 4PN order,

\footnotetext{
${ }^{3}$ The static $G^{6}$ potential at $5 \mathrm{PN}$ was recently computed in Ref. [31] (see also Ref. [32]).

${ }^{4}$ The explicit cancelation between spurious near-/far-zone divergences is not manifest in the ambiguity-free derivation within the Fokker-action approach [28,29], which instead relies on an additional worldline redefinition to remove the remaining IR/UV poles [26,27].
} 
where the cancellation of spurious divergences after the subtraction of the zero bin leaves behind physical contributions to the effective action [20]. On the one hand, it includes a term which mirrors the celebrated factor of 5/6 in the Lamb shift in QED [17,19]. On the other hand, there is also a nonlocal (in time) contribution which instead resembles the Bethe logarithm $[17,19]$. The final form of the effective action turns out to be equivalent to the one reported in Refs. [23,29], leading to the same expressions for the physical observables (such as the binding energy and periastron advance) following the careful treatment of the nonlocal term discussed in Refs. [25,27]. Our derivation thus supports the validity of the 4PN results, confirmed by three independent methodologies. At the same time, the computation within the EFT approach improves on the previous computations in Refs. [21,23-29]. Most notably, as anticipated in Refs. [17,19,20], neither ambiguity parameters nor additional regulators are required at any stage of our derivation.

The present paper is organized as follows. In Sec. II we review the EFT formalism, with an emphasis on the method of regions and IR/UV divergences with potential and radiation modes. In Sec. III we isolate the intermediate IR and UV poles in the computation of the near-zone conservative dynamics, and discuss the renormalization procedure to remove the UV divergences through counterterms. As we shall see, one of the counterterms is already fixed at third PN (3PN) order and readily removes most of the 4PN divergences. The remaining (few) UV poles are taken care of by a second counterterm, which starts at 4PN order. In Sec. IV we discuss the subtraction of the zero bin, which removes the IR singularities from the near zone. We demonstrate the explicit cancellation of the leftover UV polesuniquely determined from this procedure-against UV divergences in conservative radiation-reaction effects from the far zone. In Sec. V, after removing unphysical long- and short-distance logarithms, we present the final form of the renormalized Lagrangian, including localand nonlocal-in-time contributions. We conclude in Sec. VI with comments on the origin of the spurious IR/UV divergences. Details are relegated to the Appendices.

\section{A. Conventions}

Throughout this paper we use the following notational conventions. For spacetime variables, which depend on the proper time $\tau_{a}$, we use $v_{a}^{\alpha}\left(\tau_{a}\right) \equiv$ $\dot{x}_{a}^{\alpha}\left(\tau_{a}\right) \equiv \frac{d x_{a}^{\alpha}\left(\tau_{a}\right)}{d \tau_{a}}, \quad \dot{v}_{a}^{\alpha}\left(\tau_{a}\right)=\frac{d v_{a}^{\alpha}\left(\tau_{a}\right)}{d \tau_{a}}, \quad a_{a}^{\alpha}\left(\tau_{a}\right) \equiv \frac{D v_{a}^{\alpha}\left(\tau_{a}\right)}{D \tau_{a}} \equiv$ $\dot{v}_{a}^{\alpha}\left(\tau_{a}\right)+\Gamma_{\mu \nu}^{\alpha} v_{a}^{\mu}\left(\tau_{a}\right) v_{a}^{\nu}\left(\tau_{a}\right)$, where $x_{a}^{\mu}\left(\tau_{a}\right)$ describes the particle's worldline $(a=1,2)$. For the three-dimensional variables, which depend instead on the coordinate time $t, \quad$ we use $\quad \boldsymbol{v}_{a}(t) \equiv \dot{\boldsymbol{x}}_{a}(t) \equiv \frac{d \boldsymbol{x}_{a}(t)}{d t}, \quad \boldsymbol{a}_{a}(t) \equiv \dot{\boldsymbol{v}}_{a}(t)$, $\boldsymbol{b}_{a}(t) \equiv \dot{\boldsymbol{a}}_{a}(t), \boldsymbol{r}(t) \equiv \boldsymbol{x}_{1}(t)-\boldsymbol{x}_{2}(t), \boldsymbol{v}(t) \equiv \dot{\boldsymbol{r}}(t)=\boldsymbol{v}_{1}(t)-$ $\boldsymbol{v}_{2}(t), \quad \boldsymbol{a}(t) \equiv \dot{\boldsymbol{v}}(t)=\boldsymbol{a}_{1}(t)-\boldsymbol{a}_{2}(t), \quad$ and $\quad \boldsymbol{b}(t) \equiv \dot{\boldsymbol{a}}(t)=$ $\boldsymbol{b}_{1}(t)-\boldsymbol{b}_{2}(t)$.

\section{EFFECTIVE FIELD THEORY APPROACH}

Here we summarize the basic elements of the EFT framework put forward in Ref. [8], and further developed in Refs. [34-49], with an emphasis on the aspects discussed in Refs. [17,19,20]. For reviews of the EFT formalism applied to the binary inspiral problem, see Refs. [9-13,50,51].

\section{A. Point-particle action}

When compact bodies are probed on scales larger than their typical sizes, it is justified to write an effective theory describing a collection of worldlines (around, e.g., the center of mass of each particle) interacting with the gravitational field. The dynamics is described by an effective action:

$$
\begin{gathered}
S_{\mathrm{pp}}\left[x_{a}^{\alpha}\left(\tau_{a}\right)\right]=\sum_{a} \int d \tau_{a}\left(-m_{a}+\sum_{i} c_{i} \mathcal{O}_{i}\left[x_{a}^{\alpha}\left(\tau_{a}\right),\right.\right. \\
\left.\left.v_{a}^{\alpha}\left(\tau_{a}\right), \cdots ; g_{\mu \nu}, \partial_{\beta} g_{\mu \nu}, \cdots\right]\right) .
\end{gathered}
$$

Following the jargon of quantum field theory, we often use the term operators to denote the $\mathcal{O}_{i}$ 's. These operators are invariant under the relevant symmetries (namely, diffeomorphism and worldline reparametrizations) once on-shell conditions are imposed for the metric and matter fields.

Since we must ultimately choose a gauge when performing intermediate calculations, the need for operators that are not manifestly invariant off shell will become relevant when discussing the renormalization of the theory. For example, the operator

$$
\mathcal{O}_{a \dot{v}}=g_{\mu \nu} a^{\mu} \dot{v}^{\nu}
$$

is allowed by symmetries. Notice that this extra term vanishes on shell for nonspinning bodies, due to geodesic motion (but see footnote 26 in Sec. VI). Nevertheless, operators that are zero on shell play an important role in removing divergences which turn out to be proportional to the equations of motion. At the end of the day, they can be removed by field redefinitions. ${ }^{5}$

Other types of kinematic operators, such as $a^{\mu} a_{\mu}$, may also be added. However, in our case this operator is a "double zero" for nonspinning bodies, and therefore it

\footnotetext{
${ }^{5}$ Any term in the action proportional to the leadingorder equations of motion $D \varphi=0$, i.e., $F[\varphi] D \varphi$, where $F[\varphi]$ is some polynomial in the fields and their derivatives, can be removed by a transformation $\varphi \rightarrow \varphi-F[\varphi]$, where $\left(\boldsymbol{x}_{a}, g_{\mu \nu}\right) \in$ $\{\varphi\}$ in our case.
} 

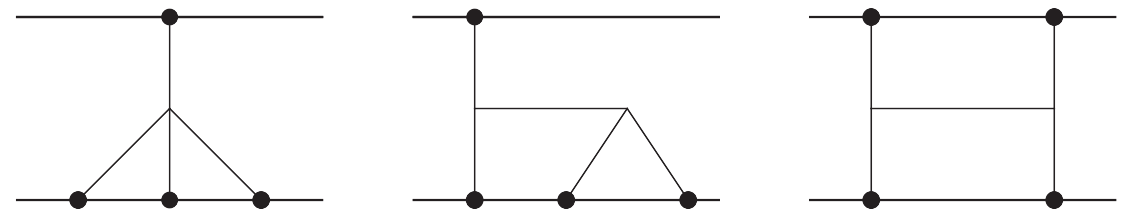

FIG. 1. Topology contributions at $\mathcal{O}\left(G^{3}\right)$ to the Lagrangian. The first two diagrams are UV divergent, starting at 3PN order, while the third diagram is finite at this order. All of these topologies are divergent at 4PN order. The first diagram is UV while the third is IR divergent. The second one has both IR and UV poles.

can be ignored. (See Refs. [52-54] for a discussion of acceleration-dependent operators that describe finite-size effects in electrodynamics.) Another class of operators, which also vanish on shell but depend on the Riemann tensor [8], are

$$
\mathcal{O}_{R} \equiv R_{\alpha}^{\alpha}, \quad \text { or } \quad \mathcal{O}_{V} \equiv R_{\mu \nu} v^{\mu} v^{\nu}
$$

where $R_{\mu \nu}$ is the Ricci tensor. The operators in Eq. (2.3) were introduced in Ref. [8] to regularize the one-point function in the static limit, while the operator in Eq. (2.2) (which is only invariant on shell) enters for nonstatic sources. While the coefficients of these particular operators are not linked with physical effects, they are required to consistently remove the UV poles in harmonic gauge.

There are, of course, physical parameters associated with finite-size terms. For instance, tidal deformations are described (at leading order) by the finite-size operator $\mathcal{O}_{E^{2}} \equiv E_{\mu \nu} E^{\mu \nu}$, where $E_{\mu \nu}$ is the electric component of the Weyl tensor. Its coefficient is often called the (electric) tidal "Love number." (A similar term may be written in terms of the magnetic component.) This operator is not relevant until fifth PN (5PN) order, and therefore does not play a role in our discussion.

\section{B. Potential region}

The EFT described by Eq. (2.1) has no reference to the PN expansion. However, once the compact bodies belong to a nonrelativistic bound state with typical separation $r$, it is useful for the purposes of manifest power counting to decompose the metric field into "potential" $\left(H_{\mu \nu}\right)$ and "radiation" $\left(\bar{h}_{\mu \nu}\right)$ modes, varying on scales $\left(k^{0},|\boldsymbol{k}|\right)_{\text {pot }} \simeq$ $(v / r, 1 / r)$ and $\left(k^{0},|\boldsymbol{k}|\right)_{\mathrm{rad}} \simeq(v / r, v / r)$, respectively, where $v$ is the relative velocity [8]. Notice that the distinction is only meaningful for $v \ll 1$, such that the perturbative expansion in the ratio of relevant scales is ultimately organized in powers of $v$. By solving for (or integrating out) the quasi-instantaneous modes order by order, one can compute the contribution to the conservative sector from potential modes. The calculation of the relevant Feynman diagrams at 4PN order was performed in Ref. [14], which we encourage the reader to consult for further details (see Appendix A for a brief summary). Because of the split into regions, spurious divergences develop in the intermediate steps. We comment below on their origin and how they are handled by the EFT approach.

\section{1. $U V$ divergences}

As it is well known, when working in a nonlinear classical theory such as general relativity, point-like sources introduce UV singularities. In dim. reg., with the number of space dimensions being $d=3+\epsilon$, the logarithmically UVdivergent integrals lead to $1 / \epsilon_{\mathrm{UV}}$ poles, as $\epsilon_{\mathrm{UV}} \rightarrow 0^{-}$. On the other hand, power-law divergences in dim. reg. are set to zero. For instance, at 3PN order the first two diagrams in Fig. 1 are logarithmically UV divergent. As discussed in Ref. [8], these classical divergences are treated as in standard quantum field theory, by writing the bare effective action in terms of a counterterm and renormalized parameters $c_{\alpha \text { bare }}=c_{\alpha, \text { c.t. }}+c_{\alpha, \text { ren }}(\mu)$, and choosing $c_{\alpha, \text { c.t. }}$ to cancel the poles to render the result finite. A renormalization scale $\mu$ is introduced in dim. reg. to account for the change in the dimensions of Newton's constant [43], e.g.,

$$
G_{d}=\mu^{3-d} G
$$

This introduces factors of $\log \mu$ in the $d \rightarrow 3$ limit when poles are present, as well as Euler's constant $\gamma_{E}$ from the expansion of the associated $\Gamma$ functions. For ease of notation in the near-zone computations we will often use the combination

$$
\bar{\mu} \equiv \mu \sqrt{4 \pi} e^{\gamma_{E} / 2}
$$

which recurrently appears in the regularization in the potential region. The $\mu$ dependence is absorbed into the renormalized parameters, rendering the results independent of the choice of renormalization scale. See Sec. V for more details.

The subtraction is of course naively ambiguous, as one is free to add any finite amount to the renormalized coefficients. For the cases when the divergence is "physical" in the sense that it does not vanish on shell, the subtraction constant is fixed by a matching procedure. Typically, this is performed by calculating response functions in external backgrounds, within the overlapping realm of validity of the full and effective theory (e.g., Refs. [55-57]). For our case, since the operators which will be needed to absorb all 


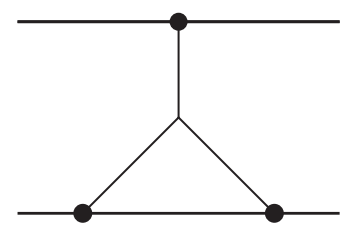

FIG. 2. The first nonlinear topology leading to IR divergences away from the static limit. The spurious poles occur when the propagators are Taylor expanded in powers of $p_{0} /|\boldsymbol{p}|$.

of the UV divergences in the near region can be removed by field redefinitions, scheme dependence on the choice of counterterms does not have a physical effect, resulting in unambiguous results.

\section{IR singularities}

Naively, IR divergences can also show up in the computation of the near-zone potential. However, since the binding is generated from modes whose wavelength is cut off by the orbital scale, any IR divergence in the near region must be spurious in nature. Ultimately, any such poles are due to the fact that we do not impose a hard cutoff on the potential modes, to avoid an explicit mutilation of diffeomorphism invariance. This is also one of the main reasons to implement dim. reg. in the intermediate calculations. As a consequence, when we perform momentum integrals in $d=3+\epsilon$ over there full range of scales we may encounter not only UV poles, but also IR singularities as $\epsilon_{\mathrm{IR}} \rightarrow 0^{+}$. For instance, IR divergences appear already in the diagram with the topology in Fig. 2 at $\mathcal{O}\left(G^{2}\right)$, with velocity corrections to the propagators of potential modes. ${ }^{6}$ Let us emphasize that these are entirely a byproduct of the splitting into regions. The IR sensitivity is induced by the quasi-instantaneous expansion of Green's functions in powers of $p^{0} /|\boldsymbol{p}|$, and therefore are not present for unexpanded propagators. See Sec. VI for more details.

Unlike UV divergences, IR singularities are due to an overlap between different regions of integration. This double counting is a well-known phenomenon in EFTs, and a systematic framework-known as the zero-bin subtraction [33] — was developed precisely to handle this issue. In the present context, the zero-bin subtraction was discussed in Refs. $[19,20]$. As we shall see, while in dim. reg. the zero-bin subtraction to $4 \mathrm{PN}$ order amounts to replacing $\epsilon_{\mathrm{IR}} \rightarrow \epsilon_{\mathrm{UV}}$ through scaleless integrals, the procedure is in principle independent of the regulator [33]. Therefore, it can also be incorporated into other formalisms, in particular to remove the ambiguity parameter(s) introduced in the derivations presented in Refs. $[23,26]$. As we demonstrate explicitly, the resulting UV poles after the

\footnotetext{
${ }^{6}$ This diagram first enters at 2PN order [39]. Further velocity corrections produce extra IR poles at higher PN orders. Other sources of IR (and UV) divergences appear in topologies at higher order in $G$, as in Fig. 1 .
}

subtraction of the zero bin cancel out against conservative contributions from the radiation region, uniquely fixing the gravitational potential.

\section{Radiation region}

After the potential modes are integrated out, the longdistance effective action for the binary system, now treated as a point-like object, is written in the form of a multipole expansion. The effective action takes the form (e.g., around the center of mass $\boldsymbol{X}$ of the binary)

$$
\begin{aligned}
S_{\mathrm{rad}}= & \int d \tau\left[-P^{\mu}(\tau) V^{\nu}(\tau)\left(1+\boldsymbol{X}^{i} \nabla_{i}\right) \bar{h}_{\mu \nu}(\tau, \boldsymbol{X}(\tau))\right. \\
& -\frac{1}{2} \bar{\omega}_{\mu}^{\alpha \beta}(\tau, \boldsymbol{X}) J_{\alpha \beta}(\tau) V^{\mu}(\tau) \\
& +\sum_{\ell=2}\left(\frac{1}{\ell !} I_{\mathrm{STF}}^{L}(\tau) \nabla_{L-2} \bar{E}_{i_{\ell-1} i_{\ell}}(\tau, \boldsymbol{X}(\tau))\right. \\
& \left.\left.-\frac{2 \ell}{(2 \ell+1) !} J_{\mathrm{STF}}^{L}(\tau) \nabla_{L-2} \bar{B}_{i_{i-1} i_{\ell}}(\tau, \boldsymbol{X}(\tau))\right)\right] .
\end{aligned}
$$

We use the shorthand notation $L \equiv\left\{i_{1} \ldots i_{\ell}\right\}$, such that $\boldsymbol{x}^{L} \equiv \boldsymbol{x}^{i_{1}} \cdots \boldsymbol{x}^{i_{\ell}}$. The four-momentum of the binary system is given by $P^{\mu}(\tau)=M V^{\mu}(\tau)$, where $V^{\mu}$ is its four-velocity and $M$ is the binding mass-energy, whereas $\omega_{\mu}^{\alpha \beta}$ are the Ricci rotation coefficients which couple to the angularmomentum tensor $J_{\alpha \beta}$. The source multipole moments $\left(I_{\mathrm{STF}}^{L}(\tau), J_{\mathrm{STF}}^{L}(\tau)\right)$ are $S O(3)$-symmetric and trace-free (STF) tensors. The bar indicates that a geometric quantity ought to be evaluated on the radiation field $\bar{h}_{\mu \nu}$.

The multipoles in Eq. (2.6) can be written in terms of moments of the stress-energy tensor using relations that rely upon the use of on-shell conservation laws [47]. This can be illustrated with the quadrupole coupling. For simplicity, let us assume that the binary is at the origin at rest, such that $\boldsymbol{X}=\dot{\boldsymbol{X}}=\mathbf{0}$. Hence, the leading term in the multipole expansion takes the form (in $d$ dimensions)

$$
\begin{aligned}
\int d t & \left(\int d^{d} \boldsymbol{x} \mathcal{T}^{i j}(t, \boldsymbol{x})\right) \bar{h}_{i j}(t, \mathbf{0}) \\
= & \int d t\left(\int d^{d} \boldsymbol{x} \mathcal{T}^{00}(t, \boldsymbol{x}) \boldsymbol{x}^{i} \boldsymbol{x}^{j}\right) \frac{1}{2} \partial_{t}^{2} \bar{h}_{i j}(t, \mathbf{0}) \\
& -\int d t\left(2 \int d^{d} \boldsymbol{x} \partial_{\mu} \mathcal{T}^{\mu i}(t, \boldsymbol{x}) \boldsymbol{x}^{j}\right) \bar{h}_{i j}(t, \mathbf{0}) \\
& -\int d t\left(\frac{1}{2} \int d^{d} \boldsymbol{x} \partial_{\mu} \partial_{\nu} \mathcal{T}^{\mu \nu}(t, \boldsymbol{x}) \boldsymbol{x}^{i} \boldsymbol{x}^{j}\right) \bar{h}_{i j}(t, \mathbf{0}) .
\end{aligned}
$$

We can then rewrite the first term as (after including other components of the metric tensor)

$$
\int d t I_{0}^{i j}(t) E_{i j}
$$




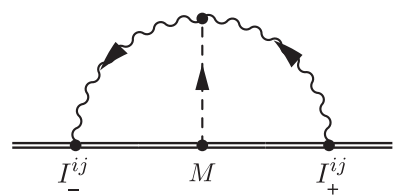

FIG. 3. Feynman diagram for the contribution to the radiationreaction force due to the tail effect. See Ref. [17] for more details.

with

$$
I_{0}^{i j}(t)=\int d^{d} \boldsymbol{x} \mathcal{T}^{00}(t, \boldsymbol{x}) \boldsymbol{x}^{i} \boldsymbol{x}^{j}
$$

at leading order in the multipole expansion. To obtain the expression in terms of the constituents of the binary we must perform a matching computation [43]; see Appendix B. The last two terms in Eq. (2.7) vanish on shell and therefore may be discarded for the derivation of physical observables. However, as we shall see, they will play an important role in canceling many of the spurious divergences arising in the potential region.

\section{Radiation reaction}

Armed with a long-distance effective theory, we can readily compute the radiation-reaction force produced by GW emission. As it was shown in Ref. [17] in the context of the EFT approach, there is both a dissipative and a conservative contribution due to GW radiation scattering off of the background geometry. To compute radiationreaction effects we use the in-in formalism adapted to a classical setting (see Refs. [58,59] for further details). At 4PN order we have the tail diagram in Fig. 3, which is UV divergent. The result in dim. reg. reads (omitting the STF label) [17]

$$
\begin{aligned}
S_{4 \mathrm{PN}}^{\mathrm{tail}}\left[\boldsymbol{x}_{a}^{ \pm}\right]= & \frac{2 G^{2} M}{5} \int_{-\infty}^{\infty} \frac{d \omega}{2 \pi} \omega^{6} I_{-}^{i j}(-\omega) I_{+}^{i j}(\omega) \\
& \times\left[-\frac{1}{\epsilon_{\mathrm{UV}}}-\gamma_{E}+\log \pi-\log \frac{\omega^{2}}{\mu^{2}}\right. \\
& \left.+\frac{41}{30}+i \pi \operatorname{sign}(\omega)\right],
\end{aligned}
$$

where

$$
\begin{aligned}
I_{-}^{i j}(t) & \equiv I^{i j}\left(t, \boldsymbol{x}_{a}^{(1)}\right)-I^{i j}\left(t, \boldsymbol{x}_{a}^{(2)}\right) \\
& =\sum_{a} m_{a}\left(\boldsymbol{x}_{a-}^{i} \boldsymbol{x}_{a+}^{j}+\boldsymbol{x}_{a+}^{i} \boldsymbol{x}_{a-}^{j}-\frac{2}{3} \delta^{i j} \boldsymbol{x}_{a-} \cdot \boldsymbol{x}_{a+}\right)+\mathcal{O}\left(\boldsymbol{x}_{a-}^{3}\right), \\
I_{+}^{i j}(t) & \equiv \frac{1}{2}\left(I^{i j}\left(t, \boldsymbol{x}_{a}^{(1)}\right)+I^{i j}\left(t, \boldsymbol{x}_{a}^{(2)}\right)\right) \\
& =\sum_{a} m_{a}\left(\boldsymbol{x}_{a+}^{i} \boldsymbol{x}_{a+}^{j}-\frac{1}{3} \delta^{i j} \boldsymbol{x}_{a+}^{2}\right)+\mathcal{O}\left(\boldsymbol{x}_{a-}^{2}\right)
\end{aligned}
$$

We will be concerned with the conservative sector in this paper, which is symmetric under $\omega \rightarrow-\omega^{7}$ The correction to the equation of motion follows from

$$
\left[\frac{\delta S_{\text {tail }}\left[\boldsymbol{x}^{a \pm}\right]}{\delta \boldsymbol{x}_{a-}}\right]_{\mathrm{PL}}=0
$$

where the "PL" subscript indicates the "physical limit" for which the "-" variables vanish and the "+" variables are set to their physical values [58]. In practice, this means that the result is half of that in Eq. (2.10) in "standard" variables, where the derivative is then allowed to hit both multipoles. From Eq. (2.12) we obtain the radiationreaction acceleration. At 4PN order, Eq. (2.10) provides an essential (and uniquely determined) contribution to the binary's dynamics similar to the Lamb shift $[17,19,20]$.

Notice that in the computation of the tail effect we have ignored multipole moments which are conserved, or terms that vanish on shell. For instance, the linear and angular momentum, as well as the extra pieces that appear following the manipulations in Eq. (2.7), have not been included. Yet, when inserted into a diagram similar to Fig. 3 these terms also produce UV-divergent integrals similar to the quadrupole contributions in Eq. (2.10). As we shall demonstrate explicitly in Sec. IV, the resulting UV poles play a key role in canceling the associated spurious singularities which develop in the potential region. As we shall see, the cancellation starts at $\mathcal{O}\left(G^{2}\right)$, and already at second PN (2PN) order, which is required for the consistency of the near-/far-zone descriptions.

\section{RENORMALIZATION OF NEAR-ZONE UV DIVERGENCES}

To renormalize the effective theory, the UV poles in the near zone must be absorbed into the counterterms in Eq. (2.1). We show here how this is implemented to 4PN order. First, we will show how to identify (when IR singularities are present) the coefficients of both UV and IR poles. This requires incorporating self-energy (scaleless) integrals. The renormalization of divergences in the potential region then proceeds without inputting information from long-distance modes, as expected. In Sec. IV we discuss the subtraction of the remaining IR singularities.

\section{A. Potential region IR/UV poles}

\section{1. $2 P N$ order}

There are no logarithmic divergences at $2 \mathrm{PN}$ order. This is the case provided we take the mass of the compact object to be time independent. However, for the sake of argument it is easy to show that if we allow for a nontrivial time

\footnotetext{
${ }^{7}$ The dissipative term $i \pi \operatorname{sign}(\omega)$ reproduces the well-known tail correction to the energy flux (e.g. Ref. [43]).
} 

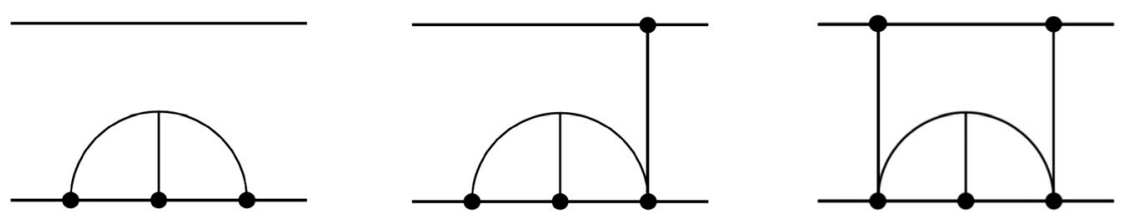

FIG. 4. Self-energy diagrams leading to scaleless integrals with logarithmic IR/UV poles at $G^{2}, G^{3}$, and $G^{4}$, respectively. Unlike the first diagram, which does not depend on the mass of the companion, the other diagrams represent self-energy corrections to the gravitational interaction.

dependence $\left[\dot{m}_{a}(t) \neq 0\right]$ the resulting $2 \mathrm{PN}$ effective Lagrangian develops logarithmic IR poles. These are due to the diagram in Fig. 2, and take the form

$$
\mathcal{L}_{2 \mathrm{PN}}^{\mathrm{IR}(\text { near })}=\frac{1}{\epsilon_{\mathrm{IR}}} G^{2}\left(\dot{m}_{1}^{2} m_{2}+2 \dot{m}_{1} \dot{m}_{2} m_{1}\right)+(1 \leftrightarrow 2) .
$$

At this stage the form of this divergence is not particularly illuminating. However, as we emphasized, to properly identify the coefficient of the IR poles in the near region we must also add a self-energy contribution. At $2 \mathrm{PN}$ order, this is represented by the first diagram in Fig. 4. The result is given by

$$
\mathcal{L}_{2 \mathrm{PN}}^{\text {self }}=\left(\frac{1}{\epsilon_{\mathrm{IR}}}-\frac{1}{\epsilon_{\mathrm{UV}}}\right) G^{2} \dot{m}_{1}^{2} m_{1}+(1 \leftrightarrow 2) .
$$

Adding the pieces together, we end up with both IR and UV poles:

$$
\mathcal{L}_{2 \mathrm{PN}}^{\mathrm{IR} / \mathrm{UV}(\text { near+self })}=\frac{1}{\epsilon_{\mathrm{IR}}} G^{2} M_{0} \dot{M}_{0}^{2}-\frac{1}{\epsilon_{\mathrm{UV}}} G^{2}\left(m_{1} \dot{m}_{1}^{2}+m_{2} \dot{m}_{2}^{2}\right) .
$$

The alert reader will immediately realize that the IR singularity now has the form of a monopole-radiation coupling proportional to $\dot{M}_{0}$, with $M_{0}=m_{1}+m_{2}$ at this order. As we have anticipated, this is consistent with the fact that this IR divergence will be associated with a contribution from the radiation modes. [The coupling to the monopole in the far zone, entering in an expression similar to Eq. (3.3), will be relevant later on.] The near-region UV divergence at this order may be absorbed into a mass renormalization. This will not be the case at higher PN orders. In what follows we will also set $\dot{m}_{a}=0$ for the divergent terms, which we kept here solely for pedagogical reasons.

\section{2. $3 P N$ order}

At this order both IR and UV divergences are present. ${ }^{8}$ Once again, the IR poles arise from the topology in Fig. 2

\footnotetext{
${ }^{8}$ In the derivation in Ref. [46], the divergences were computed in dim. reg. as poles in $(d-3)$, regardless of their IR or UV nature. Since, as we shall see, there is no physical contribution from radiation reaction at this order, this does not affect the 3PN Lagrangian. However, as we emphasized, the distinction is essential at $4 \mathrm{PN}$ order.
}

when corrections to static propagators are included, and UV divergences arise from the first two topologies in Fig. 1. The divergent terms can be recast in the following form:

$$
\begin{aligned}
\mathcal{L}_{3 \mathrm{PN}}^{\mathrm{IR} / \mathrm{UV}(\text { near })=}= & \frac{11}{3}\left(\frac{1}{\epsilon_{\mathrm{IR}}}-2 \log \bar{\mu} r\right) G^{2} m_{1}^{2} m_{2}\left(\boldsymbol{a}_{1}^{2}+2 \boldsymbol{a}_{1} \cdot \boldsymbol{a}_{2}\right) \\
& +\frac{11}{3}\left(\frac{1}{\epsilon_{\mathrm{UV}}}-3 \log \bar{\mu} r\right)\left(\frac{G^{3} m_{1}^{3} m_{2}}{r^{3}} \boldsymbol{a}_{1} \cdot \boldsymbol{r}\right) \\
& +(1 \leftrightarrow 2),
\end{aligned}
$$

where we have kept the near-zone logarithms, written in terms of $\bar{\mu}$ given in Eq. (2.5), which we will use throughout the computations. At 3PN order, the UV pole depends on the dynamical variables and therefore it cannot be simply absorbed into a mass renormalization. At the same time, the IR pole does not have the structure of a multipole moment which can be associated with computations in the long-distance theory. Both of these issues are connected. The resolution relies again on the inclusion of self-energy contributions, which are required to identify the coefficients of the IR/UV poles. The contribution from all of the logarithmically divergent scaleless integrals, given at $3 \mathrm{PN}$ order also by the topology in the first diagram of Fig. 4, yields ${ }^{9}$

$$
\begin{aligned}
& \mathcal{L}_{3 \mathrm{PN}}^{\mathrm{IR} / \mathrm{UV} \text { (self) }} \\
& =-\frac{11}{3} G^{2}\left(m_{1}^{3} \boldsymbol{a}_{1}^{2}+m_{2}^{3} \boldsymbol{a}_{2}^{2}\right)\left(\frac{(\bar{\mu} r)^{-2 \epsilon_{\mathrm{IR}}}}{\epsilon_{\mathrm{IR}}}-\frac{(\bar{\mu} r)^{-2 \epsilon_{\mathrm{UV}}}}{\epsilon_{\mathrm{UV}}}\right) .
\end{aligned}
$$

For the total result we then find

\footnotetext{
${ }^{9}$ The factors of $r^{2 e}$ account for the correct units of the worldline Lagrangian [after using Newton's constant in $d$ dimensions; see Eq. (2.4)]. The extra constants in the definition of $\bar{\mu}$ [see Eq. (2.5)], which the reader will notice cancel between the two terms, are introduced solely for convenience.
} 


$$
\begin{aligned}
\left.\mathcal{L}_{3 \mathrm{PN}}^{\mathrm{IR} / \mathrm{UV}} \text { (near+self }\right) & \\
= & -\frac{11}{3}\left(\frac{1}{\epsilon_{\mathrm{IR}}}-2 \log \bar{\mu} r\right) M_{0} G^{2}\left(m_{1} \boldsymbol{a}_{1}+m_{2} \boldsymbol{a}_{2}\right)^{2} \\
& +\frac{11}{3}\left[\left(\frac{1}{\epsilon_{\mathrm{UV}}}-2 \log \bar{\mu} r\right) G^{2} m_{1}^{3} \boldsymbol{a}_{1} \cdot\left(\boldsymbol{a}_{1}+\frac{G m_{2} \boldsymbol{r}}{r^{3}}\right)\right. \\
& \left.-\frac{G^{3} m_{1}^{3} m_{2} \boldsymbol{a}_{1} \cdot \boldsymbol{r}}{r^{3}} \log \bar{\mu} r+(1 \leftrightarrow 2)\right] .
\end{aligned}
$$

The reader will now easily notice (as we demonstrate momentarily) that the near-zone UV divergence can be removed by a counterterm whose operator vanishes on shell. (This is the case because it is proportional to the leading equation of motion: $\boldsymbol{a}_{1}+\frac{G m_{2} r}{r^{3}}=0$.) In turn, this implies that physical results are independent of the renormalization scheme. Moreover, it is also straightforward to see that the (unphysical) IR pole has the form of a dipole-radiation coupling. We will show in the next section that it is linked to a UV divergence arising from the computation of radiation-reaction effects in the long- distance EFT. In addition, we will also demonstrate how the logarithmic terms, associated to both IR and UV poles, are removed from physical quantities.

\section{4PN order}

The computation of the near-zone local-in-time gravitational potential at 4PN order was carried out in Ref. [14]. Similarly to the computations in harmonic gauge in Ref. [26], which were shown to be equivalent, the divergent terms were reported in Ref. [14] as poles in $(d-3)$, without distinguishing their IR or UV nature. However, as we have repeatedly emphasized, the proper renormalization of the effective theory relies on the correct identification of the IR and UV divergences. As we argued, this requires not only isolating the nature of the singularities in the Feynman integrals computed in Ref. [14], but also incorporating scaleless self-energy diagrams, as in Fig. 4. After adding all of the relevant diagrams including self-energy contributions, which at 4PN order also entail the second and third topologies in Fig. 4, we find

$$
\begin{aligned}
& \mathcal{L}_{G^{2}, 4 \mathrm{PN}}^{\mathrm{IR} / \mathrm{UV}(\text { near }+ \text { self })}=-G^{2} m_{1}^{2} m_{2}\left[\frac{2}{15}\left(\boldsymbol{b}_{1} \cdot \boldsymbol{r}\right)\left(\boldsymbol{b}_{2} \cdot \boldsymbol{r}\right)-\frac{19}{15} r^{2}\left(\boldsymbol{b}_{1} \cdot \boldsymbol{b}_{2}\right)+\frac{34}{15}\left(\boldsymbol{v} \cdot \boldsymbol{a}_{1}\right)\left(\boldsymbol{b}_{2} \cdot \boldsymbol{r}\right)\right. \\
& +\frac{12}{5}\left(\boldsymbol{v} \cdot \boldsymbol{a}_{2}\right)\left(\boldsymbol{b}_{1} \cdot \boldsymbol{r}\right)+\left(\boldsymbol{a}_{2} \cdot \boldsymbol{b}_{1}\right)\left(\frac{12}{5} \boldsymbol{v}_{1} \cdot \boldsymbol{r}+\frac{74}{15} \boldsymbol{v}_{2} \cdot \boldsymbol{r}\right)+\left(\frac{19}{15} \boldsymbol{a}_{2} \cdot \boldsymbol{r}+\frac{11}{6} \boldsymbol{v}^{2}\right) \boldsymbol{a}_{1}^{2} \\
& +\left(\frac{14}{3} a_{1} \cdot r-\frac{34}{15} a_{2} \cdot r+\frac{134}{15} v_{1}^{2}-\frac{16}{5} v_{1} \cdot v_{2}+\frac{79}{15} v_{2}^{2}\right)\left(a_{1} \cdot a_{2}\right)+\frac{11}{2} v_{1}^{2} a_{1}^{2} \\
& \left.+\frac{64}{5}\left(\boldsymbol{v} \cdot \boldsymbol{a}_{1}\right)\left(\boldsymbol{v} \cdot \boldsymbol{a}_{2}\right)+\frac{22}{3}\left(\boldsymbol{v}_{1} \cdot \boldsymbol{a}_{2}\right)\left(\boldsymbol{v}_{2} \cdot \boldsymbol{a}_{1}\right)+\frac{11}{3}\left(\boldsymbol{v}_{1} \cdot \boldsymbol{a}_{1}\right)^{2}\right]\left(\frac{1}{\epsilon_{\mathrm{IR}}}-2 \log \bar{\mu} r\right) \\
& -\frac{11}{3} G^{2} m_{1}^{3}\left[\left(\frac{1}{\epsilon_{\mathrm{IR}}}-2 \log \bar{\mu} r\right)-\left(\frac{1}{\epsilon_{\mathrm{UV}}}-2 \log \bar{\mu} r\right)\right]\left(\left(\boldsymbol{v}_{1} \cdot \boldsymbol{a}_{1}\right)^{2}+\frac{3}{2} \boldsymbol{v}_{1}^{2} \boldsymbol{a}_{1}^{2}\right)+(1 \leftrightarrow 2), \\
& \mathcal{L}_{G^{3}, 4 \mathrm{PN}}^{\mathrm{IR} / \mathrm{UV}(\mathrm{sear}+\mathrm{self})}=\frac{G^{3} m_{1}^{3} m_{2}}{r^{3}}\left[r^{2}\left(\frac{55}{3} \boldsymbol{a}_{1}^{2}-\frac{53}{6} \boldsymbol{a}_{1} \cdot \boldsymbol{a}_{2}\right)-\frac{11}{6}\left(\boldsymbol{a}_{1} \cdot \boldsymbol{r}\right)\left(\boldsymbol{a}_{2} \cdot \boldsymbol{r}\right)-11(\boldsymbol{v} \cdot \boldsymbol{r})\left(\boldsymbol{v} \cdot \boldsymbol{a}_{1}\right)\right. \\
& \left.+\frac{11}{3}\left(\boldsymbol{v}_{1} \cdot \boldsymbol{r}\right)\left(\boldsymbol{v}_{2} \cdot \boldsymbol{a}_{1}\right)+\left(\boldsymbol{a}_{1} \cdot \boldsymbol{r}\right)\left(\frac{22}{3} \boldsymbol{v}^{2}+\frac{11}{6} \boldsymbol{v}_{1}^{2}-\frac{11}{2} \frac{\left(\boldsymbol{v}_{2} \cdot \boldsymbol{r}\right)^{2}}{r^{2}}\right)\right]\left(\frac{1}{\epsilon_{\mathrm{UV}}}-3 \log \bar{\mu} r\right) \\
& +\frac{G^{3} m_{1}^{3} m_{2}}{r^{3}}\left[\left(\frac{124}{15} \boldsymbol{a}_{1}^{2}+\frac{10}{3} \boldsymbol{a}_{1} \cdot \boldsymbol{a}_{2}\right) r^{2}+\frac{16}{5}\left(\boldsymbol{a}_{1} \cdot \boldsymbol{r}\right)^{2}+\frac{14}{3}\left(\boldsymbol{a}_{1} \cdot \boldsymbol{r}\right)\left(\boldsymbol{a}_{2} \cdot \boldsymbol{r}\right)\right. \\
& \left.+\boldsymbol{a}_{1} \cdot \boldsymbol{r}\left(-\frac{14}{3} \boldsymbol{v}^{2}+14\left(\frac{\boldsymbol{v} \cdot \boldsymbol{r}}{r}\right)^{2}\right)-\frac{4}{3}(\boldsymbol{v} \cdot \boldsymbol{r})\left(\boldsymbol{v} \cdot \boldsymbol{a}_{1}\right)\right]\left(\frac{1}{\epsilon_{\mathrm{IR}}}-3 \log \bar{\mu} r\right) \\
& +\frac{G^{3} m_{1}^{2} m_{2}^{2}}{r^{3}}\left[\left(\frac{10}{3} \boldsymbol{a}_{1}^{2}+\frac{124}{15} \boldsymbol{a}_{1} \cdot \boldsymbol{a}_{2}\right) r^{2}+\frac{10}{3}\left(\boldsymbol{a}_{1} \cdot \boldsymbol{r}\right)^{2}+\frac{68}{15}\left(\boldsymbol{a}_{1} \cdot \boldsymbol{r}\right)\left(\boldsymbol{a}_{2} \cdot \boldsymbol{r}\right)\right. \\
& \left.-\frac{14}{3} \boldsymbol{a}_{1} \cdot \boldsymbol{r}\left(\boldsymbol{v}^{2}-3\left(\frac{\boldsymbol{v} \cdot \boldsymbol{r}}{r}\right)^{2}\right)\right]\left(\frac{1}{\epsilon_{\mathrm{IR}}}-3 \log \bar{\mu} r\right)+(1 \leftrightarrow 2) \\
& \mathcal{L}_{G^{4}, 4 \mathrm{PN}}^{\mathrm{IR} / \mathrm{UV}(\text { near }+ \text { self })}=\left(\frac{1}{\epsilon_{\mathrm{UV}}}-4 \log \bar{\mu} r\right) \frac{G^{4} m_{1} m_{2}}{3 r^{4}}\left(11 m_{1}^{2} m_{2}-23 m_{1}^{3}\right) \boldsymbol{a}_{1} \cdot \boldsymbol{r}+\left(\frac{1}{\epsilon_{\mathrm{IR}}}-4 \log \bar{\mu} r\right) \frac{4 G^{4} m_{1}^{3} m_{2}^{2}}{r^{4}} \boldsymbol{v}^{2}+(1 \leftrightarrow 2),
\end{aligned}
$$




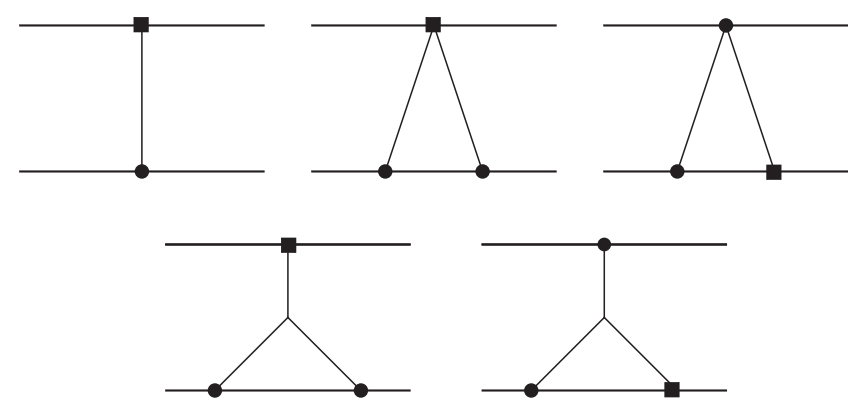

FIG. 5. Topologies for the two counterterms required to remove divergences to $4 \mathrm{PN}$ order. The black square represents an insertion of either $\mathcal{O}_{a i}$ or $\mathcal{O}_{V}$ (see text). Mirror images are also needed.

where, for future reference, we have organized the results in powers of $G .{ }^{10}$ The divergences at $\mathcal{O}\left(G^{5}\right)$ cancel out in the final result; see Refs. [18,30].

In the next section we discuss the removal the UV poles using the point-particle effective action in Eq. (2.1). This can be achieved, as expected, without knowledge from the radiation zone. We will show how to handle the IR singularities in the subsequent section, where we also discuss the contributions due to radiation modes, which are UV divergent. The main difference at 4PN order, with respect to the $2 \mathrm{PN}$ and $3 \mathrm{PN}$ cases, is the emergence (after the cancellation of these spurious IR/UV poles) of local- as well as nonlocal-in-time physical effects in the dynamics.

\section{B. UV counterterms}

\section{Operator basis}

Our task now is to remove the UV divergence from the near zone by adding the appropriate counterterm. We will use the following basis of operators in Eq. (2.1):

$$
\left\{\mathcal{O}_{a i}, \mathcal{O}_{R}, \mathcal{O}_{V}\right\}
$$

The contributions to the effective action induced by these higher-derivative terms is obtained by including new vertices in the Feynman diagrams. Notice that the first term also introduces corrections which are purely kinematic, namely, they do not depend on the variables associated with the other body. (Although, needless to say, the acceleration of each body depends on the existence of a gravitational pull induced by the companion.) The topologies needed to compute the contributions from counterterms are displayed in Fig. 5. The coefficients for these operators are chosen such that, at each PN order,

\footnotetext{
${ }^{10}$ Notice that the pole structure reported in Refs. $[14,15]$ at $\mathcal{O}\left(G^{2}\right)$ is somewhat different than the one given here. Moreover, all of the logarithmic terms are also different. This, of course, has no physical consequences and it is entirely due to the use of integration by parts and double-zero tricks.
}

$$
\mathcal{L}_{n \mathrm{PN}}^{\mathrm{UV}(\text { near+self) }}+\mathcal{L}_{n \mathrm{PN}}^{\text {c.t. }(\text { near })} \rightarrow \mathrm{UV} \text { finite. }
$$

As it was shown in Refs. [18,30], the divergences at $\mathcal{O}\left(G^{5}\right)$ cancel out at $4 \mathrm{PN}$ order. It is easy to see that $\mathcal{O}_{a i}$ does not generate a contribution at such an order in $G$. Moreover, we can also show that neither does the $\mathcal{O}_{V}$ operator. On the other hand, $\mathcal{O}_{R}$ contributes at $\mathcal{O}\left(G^{5}\right)$ in harmonic gauge. This means that it must have a finite $c_{R}$ coefficient in the $d \rightarrow 3$ limit, to avoid introducing unaccounted UV poles. Such a finite piece may be removed by a field redefinition, and therefore it plays no role in the renormalization of the theory. Hence, to deal with the UV divergences we must simply fix two parameters: $\left(c_{a i}, c_{V}\right) .{ }^{11}$ Remarkably, $\mathcal{O}_{V}$ starts to contribute at 4PN order, leaving only one of them (other than the mass) to renormalize the theory to $3 \mathrm{PN}$ order. Because of Lorentz invariance, which relates different PN orders, we shall see how determining $c_{a i}$ at $3 \mathrm{PN}$ order readily resolves higher-order divergences at $4 \mathrm{PN}$ order, up to a small mismatch which is fixed by the $c_{V}$ coefficient.

\section{Determination of $c_{a i}$}

The contribution from the $\mathcal{O}_{a i}$ operator in $d$ dimensions is obtained after expanding in the PN regime. For instance, for particle 1,

$$
\begin{aligned}
& c_{a \dot{v}} \int \mathcal{O}_{a \dot{ }} \mathrm{d} \tau \rightarrow c_{a \dot{ }}^{(1)} \int \mathrm{d} t\left[\boldsymbol{a}_{1}^{2}\left(1+\frac{3}{2} \boldsymbol{v}_{1}^{2}-\left(1+c_{d}\right) \phi\right)\right. \\
& +\left(\boldsymbol{a}_{1} \cdot \nabla \phi\right)\left(1+\frac{\left(1+c_{d}\right)}{2} \boldsymbol{v}_{1}^{2}-\phi\right) \\
& +\left(\boldsymbol{v}_{1} \cdot \boldsymbol{a}_{1}\right)\left(\left(\boldsymbol{v}_{1} \cdot \boldsymbol{a}_{1}\right)+\left(1-c_{d}\right)\left(\dot{\phi}+\boldsymbol{v}_{1} \cdot \nabla \phi\right)\right) \\
& \left.+\left(\dot{\boldsymbol{A}} \cdot \boldsymbol{a}_{1}\right)+\left(\boldsymbol{v}_{1} \cdot \nabla\right)\left(\boldsymbol{A} \cdot \boldsymbol{a}_{1}\right)-v_{1}^{i} a_{1}^{j} \nabla_{j} \boldsymbol{A}_{i}+\ldots\right],
\end{aligned}
$$

with $c_{d} \equiv \frac{2(d-1)}{(d-2)}$ and $\nabla_{i}$ represents the covariant derivative. The ellipses stand for terms involving interactions which are not relevant at $4 \mathrm{PN}$ order. We have decomposed the expression into scalar $(\phi)$, vector $(\boldsymbol{A})$, and tensor $(\sigma)$ modes; see, e.g., Ref. [18]. The correction to the Lagrangian first enters at 3PN order, and for particle 1 it takes the form

$\mathcal{L}_{3 \mathrm{PN}}^{c_{a i}^{(1)},(\text { near })}=c_{a \dot{v}}^{(1)} \boldsymbol{a}_{1} \cdot\left[\boldsymbol{a}_{1}+\frac{G m_{2} \boldsymbol{r}}{r^{3}}\left(1-\epsilon_{\mathrm{UV}}\left(\log \bar{\mu} r-\frac{3}{2}\right)\right)\right]$,

and similarly for particle 2 . The logarithmic term plus a constant piece, entering at $\mathcal{O}\left(\epsilon_{\mathrm{UV}}\right)$, are important to ensure

\footnotetext{
${ }^{11}$ This is in contrast to what was found in Ref. [8] working in background-field gauge, where $c_{R}$ and $c_{V}$ are needed to remove the poles in the one-point function. We will postpone for future work the issue of on-shell vs off-shell diffeomorphism invariance in the two-body problem, which arises due to the introduction of the $c_{a i}$ coefficient in (standard) harmonic gauge.
} 
the vanishing on shell of the $\mathcal{O}_{a i}$ operator in $d$ dimensions. Notice that only the kinematic part contributes at leading order. It is straightforward to show that the UV divergence in Eq. (3.7) can be easily removed by choosing

$$
c_{a \dot{v}, \mathrm{c.t.}}^{(a)}=-\frac{1}{\epsilon_{\mathrm{UV}}} \frac{11}{3} G^{2} m_{a}^{3} .
$$

This is in essence equivalent to the worldline shift introduced in Ref. [60]. However, the virtue of working at the level of the action is that, once their coefficients are fixed, the counterterms are determined to all orders by symmetries, in our case locality and Lorentz invariance.

\section{Determination of $c_{V}$}

Incorporating the value obtained in Eq. (3.15), the contribution from $c_{a i, \text {,.t. }}$ to the effective action at $4 \mathrm{PN}$ order then reads [see Eq. (3.13)]

$$
\begin{aligned}
\mathcal{L}_{4 \mathrm{PN}}^{\mathcal{c}_{a i, c t},(\text { near })}= & -\frac{1}{\epsilon_{\mathrm{UV}}} \frac{11}{3} G^{2} m_{1}^{3}\left(\frac{3}{2} \boldsymbol{a}_{1}^{2} \boldsymbol{v}_{1}^{2}+\left(\boldsymbol{a}_{1} \cdot \boldsymbol{v}_{1}\right)^{2}\right)-\frac{G^{3} m_{1}^{3} m_{2}}{r^{3}}\left[\frac { 1 1 } { 3 } ( \frac { 1 } { \epsilon _ { \mathrm { UV } } } - ( \operatorname { l o g } \overline { \mu } r - 1 ) ) \left(r^{2}\left(5 \boldsymbol{a}_{1}^{2}-\frac{7}{2} \boldsymbol{a}_{1} \cdot \boldsymbol{a}_{2}\right)-\frac{1}{2}\left(\boldsymbol{a}_{1} \cdot \boldsymbol{r}\right)\left(\boldsymbol{a}_{2} \cdot \boldsymbol{r}\right)\right.\right. \\
& \left.+\left(\boldsymbol{v}_{1} \cdot \boldsymbol{r}\right)\left(\boldsymbol{v}_{2} \cdot \boldsymbol{a}_{1}\right)+\left(\boldsymbol{a}_{1} \cdot \boldsymbol{r}\right)\left(2 \boldsymbol{v}^{2}+\frac{1}{2} \boldsymbol{v}_{1}^{2}-\frac{3\left(\boldsymbol{v}_{2} \cdot \boldsymbol{r}\right)^{2}}{2}\right)-3(\boldsymbol{v} \cdot \boldsymbol{r})\left(\boldsymbol{v} \cdot \boldsymbol{a}_{1}\right)\right) \\
& \left.+r^{2}\left(\frac{15}{4} \boldsymbol{a}_{1} \cdot \boldsymbol{a}_{2}-\frac{9}{2} \boldsymbol{a}_{1}^{2}\right)-\frac{1}{4}\left(\boldsymbol{a}_{1} \cdot \boldsymbol{r}\right)\left(\boldsymbol{a}_{1} \cdot \boldsymbol{r}\right)+\left(\boldsymbol{a}_{1} \cdot \boldsymbol{r}\right)\left(\frac{1}{4} \boldsymbol{v}_{1}^{2}-\frac{5}{4}\left(\frac{\boldsymbol{v}_{2} \cdot \boldsymbol{r}}{r}\right)^{2}\right)+\frac{1}{2}(\boldsymbol{v} \cdot \boldsymbol{r})\left(\boldsymbol{v}_{1} \cdot \boldsymbol{a}_{1}\right)+\frac{1}{2}\left(\boldsymbol{v}_{2} \cdot \boldsymbol{r}\right)\left(\boldsymbol{v}_{2} \cdot \boldsymbol{a}_{1}\right)\right] \\
& +\frac{11}{3}\left(\frac{1}{\epsilon_{\mathrm{UV}}}-2(\log \bar{\mu} r-1)\right) \frac{G^{4} m_{1}^{3} m_{2}\left(m_{1}-m_{2}\right)}{r^{4}}\left(\boldsymbol{a}_{1} \cdot \boldsymbol{r}\right)+(1 \leftrightarrow 2) .
\end{aligned}
$$

The above expression already removes many of the UV divergences in Eqs. (3.8)-(3.10). It is then straightforward to show that the remaining UV poles can be absorbed into the $\mathcal{O}_{V}$ operator. The contribution to the effective action takes the form

$$
\begin{aligned}
\mathcal{L}_{4 \mathrm{PN}}^{c_{V},(\text { near })}= & 4 c_{V}^{(1)}\left(-\boldsymbol{a}_{1} \cdot \boldsymbol{a}_{2} \frac{G m_{2}}{r}\left(1-\epsilon_{\mathrm{UV}} \log \bar{\mu} r\right)\right. \\
& \left.+\left(\boldsymbol{a}_{1} \cdot \boldsymbol{r}\right) \frac{G^{2} m_{1} m_{2}}{r^{4}}\left[1-2 \epsilon_{\mathrm{UV}}\left(\log \bar{\mu} r-\frac{3}{4}\right)\right]\right) \\
& +(1 \leftrightarrow 2),
\end{aligned}
$$

such that choosing the counterterm

$$
c_{V, \text { c.t. }}^{(a)}=\frac{1}{\epsilon_{\mathrm{UV}}} G^{2} m_{a}^{3}
$$

removes the remaining UV poles. In summary, the (bare) point-particle effective action in Eq. (2.1) becomes ${ }^{12}$

$$
\begin{aligned}
& S_{\mathrm{pp}}\left[x_{a}^{\alpha}\left(\tau_{a}\right)\right] \\
& =\sum_{a} \int d \tau_{a}\left[-m_{a}+\left(c_{a \dot{r}, \mathrm{ren}}^{(a)}(\mu)-\frac{11}{3} \frac{G^{2} m_{a}^{2}}{\epsilon_{\mathrm{UV}}}\right) g_{\mu \nu} a_{a}^{\mu} \dot{v}_{a}^{\nu}\right. \\
& \left.\quad+\left(c_{V, \text { ren }}^{(a)}(\mu)+\frac{G^{2} m_{a}^{2}}{\epsilon_{\mathrm{UV}}}\right) R_{\mu \nu} v_{a}^{\mu} v_{a}^{\nu}\right] .
\end{aligned}
$$

\footnotetext{
${ }^{12}$ Notice that the rather simple expression in Eq. (3.19) predicts much of the structure of UV poles that will also appear at higher PN orders. In contrast, in the Fokker-action treatment the removal of the UV divergences is performed, independently at each PN order, through an ad hoc worldline shift [26].
}

This completes the UV renormalization of the theory to 4PN order.

While we have chosen an MS scheme in Eqs. (3.15) and (3.18) to remove the poles, there is always a degree of ambiguity in the choice of counterterms. (For instance, we could choose the $\overline{\mathrm{MS}}$ scheme and remove the extra constants we kept in the definition of $\bar{\mu}$.) This is often resolved by a matching computation, as we alluded to before. However, as we discussed, all of the operators in Eq. (3.11) can be removed by field redefinitions. This means that shifts in the counterterms or renormalized parameters have no physical effect. We will return in Sec. V to address this remaining freedom in the effective theory. As we shall see, the renormalized coefficients can be used to remove unphysical UV logarithms (as well as factors of $\mu$ ) from the final expressions.

\section{CANCELLATION OF NEAR-/FAR-ZONE IR/UV DIVERGENCES}

After the near-zone UV divergences are removed by counterterms, the remaining task is to deal with the IR singularities. As we discussed, this is done by implementing the zero-bin subtraction $[19,20]$. In practice, the subtraction of the zero bin transforms IR poles into UV poles [see Eq. (4.3) below]. The main reason is due to the type of integrals that enter at this order, such that the zero bin appears as a scaleless contribution [20]. Once the IR poles turn into UV divergences, they cancel out against UV poles arising in conservative contributions from the far zone. The divergences appear in the computation of the tail effect in the long-distance effective theory, in which the 
binary itself is treated as a point-like source endowed with a series of multipole moments. While the subtraction of the zero bin may seem like a simple formal manipulation in dim. reg., it unambiguously determines all of the finite pieces in the gravitational potential. Moreover, the procedure is regularization independent [33], and therefore it may be used to remove the ambiguities plaguing other derivations. As we demonstrate, the cancellation between spurious divergences is already at work at $2 \mathrm{PN}$ and $3 \mathrm{PN}$ orders. Starting at 4PN order, tail terms from the radiation region begin to contribute to the conservative dynamics.

\section{A. Zero-bin IR subtraction}

The IR divergences that we encounter when computing in the potential region can be ultimately traced back to the master integral (similar to the so-called "Riesz formula" in $d$ dimensions [7])

$$
\begin{aligned}
I\left[n_{1}, n_{2}\right] \equiv & \int_{\boldsymbol{k}} \frac{1}{\left[\boldsymbol{k}^{2}\right]^{n_{1}\left[(\boldsymbol{k}+\boldsymbol{p})^{2}\right]^{n_{2}}}} \\
= & \frac{\Gamma\left[n_{1}+n_{2}-d / 2\right] \Gamma\left[d / 2-n_{2}\right] \Gamma\left[d / 2-n_{1}\right]}{(4 \pi)^{d / 2} \Gamma\left[n_{1}\right] \Gamma\left[n_{2}\right] \Gamma\left[d-n_{1}-n_{2}\right]} \\
& \times\left(\boldsymbol{p}^{2}\right)^{d / 2-n_{1}-n_{2}},
\end{aligned}
$$

where $\int_{k} \equiv \int \frac{d^{d} k}{(2 \pi)^{d}}$; see Appendix A. The IR divergence is manifest by the fact that the right-hand side can become singular for $d<3$. As discussed in Ref. [20], the implementation of the zero-bin subtraction is straightforward in this case. The singular term comes from the region of integration where the momenta is soft, $\boldsymbol{k} \ll \boldsymbol{p}$, which must be subtracted away. For instance, the pair $\left(n_{1}=3 / 2, n_{2}=1 / 2\right)$ occurs repeatedly. In that case, by expanding the factor of $(\boldsymbol{k}+\boldsymbol{p})$ the zero-bin contribution becomes ${ }^{13}$ [20]

$$
\begin{aligned}
I_{\mathrm{ZB}}\left[n_{1}, n_{2}\right] & =\int_{\boldsymbol{k}} \frac{1}{\left[\boldsymbol{k}^{2}\right]^{n_{1}}\left[\boldsymbol{p}^{2}\right]^{n_{2}}} \stackrel{\left(n_{1}=3 / 2, n_{2}=1 / 2\right)}{\longrightarrow}|\boldsymbol{p}|^{-1} \int_{\boldsymbol{k}} \frac{1}{\boldsymbol{k}^{3}} \\
& =\frac{i}{16 \pi|\boldsymbol{p}|}\left(\frac{1}{\epsilon_{\mathrm{UV}}}-\frac{1}{\epsilon_{\mathrm{IR}}}\right) .
\end{aligned}
$$

It is now straightforward to implement the subtraction in all of the IR divergent Feynman integrals to 4PN order, including the self-energy contributions, which themselves are scalelesstype integrals. At the end of the day, after removing the zero bin, in practice the IR poles turn into UV divergences according to the replacement, for $n \leq 4$,

$$
\left.\mathcal{L}_{n \mathrm{PN}}^{\mathrm{UV}(\mathrm{IR} \text { near }+ \text { self-ZB })} \equiv \mathcal{L}_{n \mathrm{PN}}^{\mathrm{IR}(\text { near }+ \text { self })}\right|_{\epsilon_{\mathrm{IR}} \rightarrow \epsilon_{\mathrm{UV}}} .
$$

Let us stress two important points. First of all, while the subtraction of the zero bin changes the nature of the pole, crucially it does not introduce any extra finite pieces at this

\footnotetext{
${ }^{13}$ In $d$ dimensions, the (scaleless) zero-bin integral(s) also includes the additional factors displayed in, e.g., Eq. (3.6).
}

order. This feature, which is only true in dim. reg., is also due to the type of integrals that contribute to $4 \mathrm{PN}$ order. Second, the UV poles from the scaleless integrals have been absorbed into counterterms, as expected. Therefore, there are leftover IR divergences from self-energies in the near zone, which turn into UV poles after the zero bin is removed from the scaleless integrals. In what follows we demonstrate how the leftover UV poles, after the subtraction of the zero bin, explicitly cancel out against counterparts arising from conservative contributions in the far zone. ${ }^{14}$

\section{B. Radiation-reaction UV poles}

In the calculation of the tail contribution yielding Eq. (2.10) at 4PN order, only the physical quadrupole coupling in Eq. (2.6) was retained. However, as we emphasized, other types of couplings (e.g., the dipole term) may induce UV divergences in the far zone, albeit without leading to a physical effect. Below we compute all of the UV singularities which appear in the EFT computation of the conservative radiation-reaction tail effects, including those which vanish on shell. These will be essential for removing the UV poles remaining in the near region after the zero-bin subtraction. For simplicity, all of the results quoted below are given in standard (as opposed to \pm ) variables, such that the variation of the action follows the usual steps.

\section{1. $2 P N$}

At 2PN order we encounter a diagram similar to Fig. 3, but instead of the quadrupole moment we insert the monopole term $\int M(\tau) d \tau$ [see Eq. (2.6)]. Keeping only the conservative part, we find

$$
\begin{aligned}
& \int d t \mathcal{L}_{2 \mathrm{PN}}^{\mathrm{UV}(\mathrm{far})} \\
& =-i \frac{M_{0}}{M_{\mathrm{Pl}}^{2}} \int_{\boldsymbol{k}, \boldsymbol{q}} \int \frac{d \omega}{2 \pi}|M(\omega)|^{2}\langle\phi(\omega, \boldsymbol{q}) \phi(\omega, \boldsymbol{q}-\boldsymbol{k}) \phi(0, \boldsymbol{q})\rangle_{\mathrm{UV}} \\
& =-\frac{G^{2} M_{0}}{\epsilon_{\mathrm{UV}}} \int \frac{d \omega}{2 \pi} \omega^{2}|M(\omega)|^{2}\left(1+\epsilon_{\mathrm{UV}} \log \left(\frac{4 \omega^{2} e^{2 \gamma_{E}}}{\bar{\mu}^{2}}\right)\right)
\end{aligned}
$$

where, in order to illustrate the cancellation against the near-zone logarithms we have written the result in terms of $\bar{\mu}$ in Eq. (2.5) (but omitted other constants), and already transformed from \pm into standard variables. While we have taken the leading-order part of the background

\footnotetext{
${ }^{14}$ Note that algebraically, if applied before the UV counterterms, the zero-bin subtraction would cancel self-energy terms exactly. Of course, at the end of the day the remaining (artificial) UV poles in the near and far zones would also vanish in the final results. However, the procedure outlined here-working with Eq. (3.19) to renormalize the near-zone effective theory with potential modes before implementing the zero-bin subtractionclarifies the nature of the spurious poles and demonstrates the mutual cancellation. See Sec. VI for more details.
} 
geometry proportional to $M_{0}$, we have allowed for higherorder PN corrections to the monopole coupling, i.e., $M(t)=M_{0}+\cdots$, which will play a key role later on.

The reader will immediately notice that the UV pole from the far zone has (minus) the coefficient of the IR counterpart at 2PN order in Eq. (3.3). Hence, following the zero-bin subtraction, we thus arrive at

$$
\begin{aligned}
& \int d t\left(\mathcal{L}_{2 \mathrm{PN}}^{\mathrm{UV}(\mathrm{IR} \text { near+self-ZB })}+\mathcal{L}_{2 \mathrm{PN}}^{\mathrm{UV}(\mathrm{far})}\right) \\
& \rightarrow-G^{2} M_{0} \int \frac{d \omega}{2 \pi} \omega^{2}|M(\omega)|^{2} \log \left(4 \omega^{2} e^{2 \gamma_{E}}\right) .
\end{aligned}
$$

Needless to say, since $\dot{M}=0$ on shell, all of these manipulations involving the monopole coupling do not contribute to anything physical. However, we have already started to reveal the pattern that will continue to appear at higher PN orders.

\section{2. $3 P N$}

Next, at 3PN order we have corrections from the monopole term as well as contributions from the dipole coupling. The result can be split into two parts. While the effects due to the background geometry remain as the (leading-order) scalar exchange, there are now scalar $\left(\phi^{3}\right)$ and vector $\left(A^{2} \phi\right)$ couplings. The computation is straightforward and, using $\boldsymbol{P}=M_{0} \dot{\boldsymbol{X}}$ at leading order, we find

$$
\begin{aligned}
& \int d t \mathcal{L}_{\phi^{3}, 3 \mathrm{PN}}^{\mathrm{UV}(\mathrm{far})} \\
& =-\frac{G^{2} M_{0}^{3}}{3 \epsilon_{\mathrm{UV}}} \int \frac{d \omega}{2 \pi} \omega^{4}|\boldsymbol{X}(\omega)|^{2}\left(\frac{1}{\epsilon_{\mathrm{UV}}}+\log \left(\frac{4 \omega^{2} e^{2 \gamma_{E}}}{\bar{\mu}^{2}}\right)\right), \\
& \int d t \mathcal{L}_{\phi A^{2}, 3 \mathrm{PN}}^{\mathrm{UV}(\mathrm{fr})} \\
& =\frac{4 G^{2} M_{0}^{3}}{\epsilon_{\mathrm{UV}}} \int \frac{d \omega}{2 \pi} \omega^{4}|\boldsymbol{X}(\omega)|^{2}\left(\frac{1}{\epsilon_{\mathrm{UV}}}+\log \left(\frac{4 \omega^{2} e^{2 \gamma_{E}}}{\bar{\mu}^{2}}\right)\right),
\end{aligned}
$$

where as before we have written the associated logarithmic contribution in terms of $\bar{\mu}$. The two terms combined lead to

$$
\begin{aligned}
\int d t \mathcal{L}_{3 \mathrm{PN}}^{\mathrm{UV}(\text { far })}= & \frac{11}{3} M_{0}^{3} G^{2} \int \frac{d \omega}{2 \pi} \omega^{4}|\boldsymbol{X}(\omega)|^{2} \\
& \times\left(\frac{1}{\epsilon_{\mathrm{UV}}}+\log \left(\frac{4 \omega^{2} e^{2 \gamma_{E}}}{\bar{\mu}^{2}}\right)\right) .
\end{aligned}
$$

Once again the reader will identify the coefficient of the far-zone UV divergence with (minus) the one in the IR pole from the near region in Eq. (3.7), after noticing $\ddot{\boldsymbol{X}}=m_{1} \boldsymbol{a}_{1}+m_{2} \boldsymbol{a}_{2}$, at leading order. The cancellation of divergences (as well as the associated $\log \bar{\mu}$ 's) follows,

$$
\begin{aligned}
& \int d t\left(\mathcal{L}_{3 \mathrm{PN}}^{\mathrm{UV}(\mathrm{IR} \text { near+self-ZB })}+\mathcal{L}_{3 \mathrm{PN}}^{\mathrm{UV}(\text { far })}\right) \\
& \rightarrow \frac{11}{3} M_{0}^{3} G^{2} \int \frac{d \omega}{2 \pi} \omega^{4}|\boldsymbol{X}(\omega)|^{2} \log \left(4 \omega^{2} e^{2 \gamma_{E}}\right),
\end{aligned}
$$

as anticipated. The reader will notice that, at 3PN order, the remaining logarithm multiplies a term which is a double zero on shell, and therefore it can be ignored. This will not be the case at $4 \mathrm{PN}$ order.

The previous examples illustrate how, once the poles are identified and the zero-bin subtraction implemented, the IR/UV spurious divergences and associated $\log \mu$ 's cancel each other out between near- and far-zone contributions. Of course, there are no leftover finite terms to 3PN order from this procedure, since these all vanish on shell. We find the first nontrivial (finite) contribution to the conservative sector from the far zone at 4PN order. The cancellation of divergences, in any case, proceeds in a similar fashion.

\section{3. $4 P N$}

The contribution from the quadrupole coupling $I^{i j} E_{i j}$ was computed before [see Eq. (2.10)]. After plugging the form of $I_{0}^{i j}$ at leading order, we find

$$
S_{4 \mathrm{PN}, M\left(I_{0}^{i j}\right)^{2}}^{\text {tail (far) }}=S_{4 \mathrm{PN}}^{\text {tail (loc) }}+S_{4 \mathrm{PN}}^{\text {tail (nonloc) }},
$$

where we have isolated the local-in-time contribution ${ }^{15}$

$$
\begin{aligned}
S_{4 \mathrm{PN}}^{\mathrm{tail}(\mathrm{loc})}= & -\frac{G^{2} M_{0}^{3} \nu^{2}}{5} \int d t\left\{\left(\frac{1}{\epsilon_{\mathrm{UV}}}-2 \log \bar{\mu} r-\frac{41}{30}\right)\right. \\
& \times\left(2 r^{2} \boldsymbol{b}^{2}+\frac{2}{3}(\boldsymbol{r} \cdot \boldsymbol{b})^{2}+18 \boldsymbol{a}^{2} \boldsymbol{v}^{2}+6(\boldsymbol{v} \cdot \boldsymbol{a})^{2}\right. \\
& -8(\boldsymbol{r} \cdot \boldsymbol{b})(\boldsymbol{v} \cdot \boldsymbol{a})+12(\boldsymbol{a} \cdot \boldsymbol{b})(\boldsymbol{r} \cdot \boldsymbol{v}) \\
& +12(\boldsymbol{v} \cdot \boldsymbol{b})(\boldsymbol{r} \cdot \boldsymbol{a}))+\frac{4}{9}(\boldsymbol{r} \cdot \boldsymbol{b})^{2}+4(\boldsymbol{v} \cdot \boldsymbol{a})^{2} \\
& \left.+\frac{8}{3}(\boldsymbol{r} \cdot \boldsymbol{b})(\boldsymbol{v} \cdot \boldsymbol{a})\right\}
\end{aligned}
$$

(where $\nu \equiv \frac{m_{1} m_{2}}{M_{0}^{2}}$ is the symmetric mass ratio), and nonlocalin-time contribution

$$
\begin{aligned}
S_{4 \mathrm{PN}}^{\text {tail(nonloc) }} & \equiv \int d t \mathcal{L}_{4 \mathrm{PN}}^{G^{2} \log v} \\
& =-\frac{G^{2} M_{0}}{5} \int_{-\infty}^{\infty} \frac{d \omega}{2 \pi} \omega^{6}\left|I^{i j}(\omega)\right|^{2} \log \left(4 \omega^{2} r^{2} \mathrm{e}^{2 \gamma_{E}}\right)
\end{aligned}
$$

\footnotetext{
${ }^{15}$ The terms in the third line originate from $\mathcal{O}\left(\epsilon_{\mathrm{UV}}\right)$ corrections to the quadrupole in $d$ dimensions hitting the UV pole. These terms play a key role in deriving the correct physical expression in the $d \rightarrow 3$ limit.
} 
to the effective action. The nonlocal-in-time part was obtained earlier in Ref. [61], and emphasized more recently in Ref. [23]. In order to perform the above splitting, we have separated the $\log \mu$ from Eq. (2.10) (which we rewrote in terms of $\bar{\mu}$ ) and $\log \omega$, and introduced (by hand) the factors of $\log r{ }^{16}$ This is simply for convenience, so that in this fashion the factor of $\log \bar{\mu} r$ cancels out against a logarithmic contribution from the near zone. ${ }^{17}$

Notice that, without using the equations of motion, the UV pole enters at $\mathcal{O}\left(G^{2}\right)$. Hence, it is clearly not sufficient to cancel against the IR poles from the near region in Eqs. (3.8)-(3.10) after the zero-bin subtraction. Moreover, the coefficients of the $G^{2}$ divergences do not match either. As we demonstrate in what follows, the reason for the mismatch is due to contributions from the long-distance EFT which we have ignored thus far, since they vanish on shell; see Eq. (2.7). However, the additional terms are needed to remove all of the spurious IR/UV divergences. The extra terms in the effective action that result from the multipole expansion in the far zone, but do not contribute to physical quantities, can be written as follows:

$$
\Delta S_{\mathrm{rad}}=\frac{1}{M_{\mathrm{Pl}}} \int d t\left[\frac{1}{2}\left(T^{i j}-\frac{I^{i j(2)}}{2}\right) \bar{h}_{i j}+\frac{1}{2}\left(\left(M^{i j}+M^{j i}\right)-I^{i j(1)}\right) \bar{h}_{0 i, j}+\cdots\right] .
$$

We use the superscript $(n)$ to indicate $n$ time derivatives. The moments of the pseudo-stress-energy tensor that appear in the above expression (other than the usual suspects) are given by

$$
T^{i j}(t) \equiv \int d^{3} \boldsymbol{x} \mathcal{T}^{i j}(t, \boldsymbol{x}), \quad M^{i(L-1)}(t) \equiv \int d^{3} \boldsymbol{x} \mathcal{T}^{0 i}(t, \boldsymbol{x}) \boldsymbol{x}^{L-1}
$$

Notice that, as expected, the coefficients in Eq. (4.13) vanish once conservation laws are enforced [e.g., Eq. (2.7)]. Yet, each of these terms induces extra (divergent) contributions to the tail effect in radiation reaction. More explicitly, the expression in Eq. (4.13) gives rise to terms which can be written in a generic form as follows:

$$
\mathcal{L}_{4 \mathrm{PN}, \Delta S_{\mathrm{rad}}}^{\mathrm{tail}(\mathrm{far})}=G^{2} \int_{-\infty}^{\infty} \frac{d \omega}{2 \pi} \omega^{6} \sum_{i} \mathcal{M}_{i}(\omega)\left[\frac{1}{\epsilon_{\mathrm{UV}}}-2 \log \bar{\mu} r+\log \left(4 \omega^{2} r^{2} \mathrm{e}^{2 \gamma_{E}}\right)+\cdots\right]
$$

where the ellipses include finite terms [similarly to the celebrated $41 / 30$ in Eq. (2.10)] and the $\log \bar{\mu} r$ [as in Eq. (4.10)] is introduced for convenience. Unlike the contribution from Eq. (4.10), each of the $\mathcal{M}_{i}(\omega)$ 's vanishes on shell in $d$ dimensions.

The computation of additional tail terms is lengthy but straightforward; see Appendix B. After gathering all of the pieces, the resulting UV poles and logarithmic terms take the form

$$
\begin{aligned}
\mathcal{L}_{G^{2}, 4 \mathrm{PN}}^{\mathrm{UV}(\mathrm{far})}= & G^{2} m_{1}^{2} m_{2}\left[\frac{2}{15}\left(\boldsymbol{b}_{1} \cdot \boldsymbol{r}\right)\left(\boldsymbol{b}_{2} \cdot \boldsymbol{r}\right)-\frac{19}{15} r^{2}\left(\boldsymbol{b}_{1} \cdot \boldsymbol{b}_{2}\right)+\frac{34}{15}\left(\boldsymbol{v} \cdot \boldsymbol{a}_{1}\right)\left(\boldsymbol{b}_{2} \cdot \boldsymbol{r}\right)+\frac{12}{5}\left(\boldsymbol{v} \cdot \boldsymbol{a}_{2}\right)\left(\boldsymbol{b}_{1} \cdot \boldsymbol{r}\right)+\left(\boldsymbol{a}_{2} \cdot \boldsymbol{b}_{1}\right)\left(\frac{12}{5} \boldsymbol{v}_{1} \cdot \boldsymbol{r}+\frac{74}{15} \boldsymbol{v}_{2} \cdot \boldsymbol{r}\right)\right. \\
& +\left(\frac{19}{15} \boldsymbol{a}_{2} \cdot \boldsymbol{r}+\frac{11}{6} \boldsymbol{v}^{2}\right) \boldsymbol{a}_{1}^{2}+\left(\frac{14}{3} \boldsymbol{a}_{1} \cdot \boldsymbol{r}-\frac{34}{15} \boldsymbol{a}_{2} \cdot \boldsymbol{r}+\frac{134}{15} \boldsymbol{v}_{1}^{2}-\frac{16}{5} \boldsymbol{v}_{1} \cdot \boldsymbol{v}_{2}+\frac{79}{15} \boldsymbol{v}_{2}^{2}\right)\left(\boldsymbol{a}_{1} \cdot \boldsymbol{a}_{2}\right)+\frac{64}{5}\left(\boldsymbol{v} \cdot \boldsymbol{a}_{1}\right)\left(\boldsymbol{v} \cdot \boldsymbol{a}_{2}\right) \\
& \left.+\frac{22}{3}\left(\boldsymbol{v}_{1} \cdot \boldsymbol{a}_{2}\right)\left(\boldsymbol{v}_{2} \cdot \boldsymbol{a}_{1}\right)+\frac{11}{3}\left(\boldsymbol{v}_{1} \cdot \boldsymbol{a}_{1}\right)^{2}+\frac{11}{2} \boldsymbol{v}_{1}^{2} \boldsymbol{a}_{1}^{2}\right]\left(\frac{1}{\epsilon_{\mathrm{UV}}}-2 \log \bar{\mu} r\right) \\
& +\frac{11}{3} G^{2} m_{1}^{3}\left(\left(\boldsymbol{v}_{1} \cdot \boldsymbol{a}_{1}\right)^{2}+\frac{3}{2} \boldsymbol{v}_{1}^{2} \boldsymbol{a}_{1}^{2}\right)\left(\frac{1}{\epsilon_{\mathrm{UV}}}-2 \log \bar{\mu} r\right)+(1 \leftrightarrow 2)
\end{aligned}
$$

\footnotetext{
${ }^{16}$ For the sake of simplicity, in writing Eq. (4.12) we used an abuse of notation where the "mixed term" $\int \frac{d \omega}{2 \pi} \omega^{6}\left|I^{i j}(\omega)\right|^{2} \log r$ replaces the correct expression $\int d t I^{i j(3)}(t) I^{i j(3)}(t) \log r(t)$. The superscript (3) indicates three time derivatives.

${ }^{17}$ Notice that the cancellation leaves behind finite terms, associated with regularization-dependent constants, i.e., $(\bar{\mu} / \mu)^{2}=4 \pi e^{\gamma_{E}}$. This mismatch is behind the factor of $\log \left(16 e^{2 \gamma_{E}}\right)$, in addition to the $\log x$ [with $x \equiv(G M \omega)^{2 / 3}$ ], in the expression for the binding energy in a circular orbit at 4PN order; see Ref. [23].
} 


$$
\begin{gathered}
\mathcal{L}_{G^{3}, 4 \mathrm{PN}}^{\mathrm{UV}(\mathrm{far})}=-\frac{G^{3} m_{1}^{3} m_{2}}{r^{3}}\left[\left(\frac{124}{15} \boldsymbol{a}_{1}^{2}+\frac{10}{3} \boldsymbol{a}_{1} \cdot \boldsymbol{a}_{2}\right) r^{2}+\frac{16}{5}\left(\boldsymbol{a}_{1} \cdot \boldsymbol{r}\right)^{2}+\frac{14}{3}\left(\boldsymbol{a}_{1} \cdot \boldsymbol{r}\right)\left(\boldsymbol{a}_{2} \cdot \boldsymbol{r}\right)+\boldsymbol{a}_{1} \cdot \boldsymbol{r}\left(-\frac{14}{3} \boldsymbol{v}^{2}+14\left(\frac{\boldsymbol{v} \cdot \boldsymbol{r}}{\boldsymbol{r}}\right)^{2}\right)\right. \\
\left.-\frac{4}{3}(\boldsymbol{v} \cdot \boldsymbol{r})\left(\boldsymbol{v} \cdot \boldsymbol{a}_{1}\right)\right]\left(\frac{1}{\epsilon_{\mathrm{UV}}}-3 \log \bar{\mu} r\right)-\frac{G^{3} m_{1}^{2} m_{2}^{2}}{r^{3}}\left[\left(\frac{10}{3} \boldsymbol{a}_{1}^{2}+\frac{124}{15} \boldsymbol{a}_{1} \cdot \boldsymbol{a}_{2}\right) r^{2}+\frac{10}{3}\left(\boldsymbol{a}_{1} \cdot \boldsymbol{r}\right)^{2}+\frac{68}{15}\left(\boldsymbol{a}_{1} \cdot \boldsymbol{r}\right)\left(\boldsymbol{a}_{2} \cdot \boldsymbol{r}\right)\right. \\
\left.-\frac{14}{3} \boldsymbol{a}_{1} \cdot \boldsymbol{r}\left(\boldsymbol{v}^{2}-3\left(\frac{\boldsymbol{v} \cdot \boldsymbol{r}}{r}\right)^{2}\right)\right]\left(\frac{1}{\epsilon_{\mathrm{UV}}}-3 \log \bar{\mu} r\right)+(1 \leftrightarrow 2) \\
\mathcal{L}_{G^{4}, 4 \mathrm{PN}}^{\mathrm{UV}(\mathrm{far})}=-\left(\frac{1}{\epsilon_{\mathrm{UV}}}-4 \log \bar{\mu} r\right) \frac{4 G^{4} m_{1}^{3} m_{2}^{2}}{r^{4}} \boldsymbol{v}^{2}+(1 \leftrightarrow 2)
\end{gathered}
$$

where we performed the manipulations to introduce the factors of $\log r$ described after Eq. (4.12) and, for notational simplicity, we did not include the leftover $\log \omega r$ 's (see below). The cancellation between the near- and far-zone divergences as well as $\log \bar{\mu} r$ 's is now evident,

$$
\int d t\left(\mathcal{L}_{4 \mathrm{PN}}^{\mathrm{UV}}{ }^{(\mathrm{IR} \text { near }+ \text { self-ZB })}+\mathcal{L}_{4 \mathrm{PN}}^{\mathrm{UV}}{ }^{(\text {far })}\right) \rightarrow \int \frac{d \omega}{2 \pi}(\cdots) \times \log \left(4 \omega^{2} r^{2} e^{2 \gamma_{E}}\right),
$$

similarly to the 3PN case in Eq. (4.9). The ellipses represents a series of finite terms at each order in $G$. The result thus includes long-distance logarithms, of which only the one in Eq. (4.12) enters in the conservative dynamics.

It is important to notice that, even though they do not play a role in the cancellation of intermediate divergences, there are other extra finite pieces resulting from the terms displayed in Eq. (4.15) that are required to ensure the cancellation of unphysical (finite) contributions. These terms arise, as in Eq. (4.10), from the UV poles hitting the $\mathcal{O}\left(\epsilon_{\mathrm{UV}}\right)$ corrections in the (vanishing) $d$-dimensional multipole moments $\left[\mathcal{M}_{i}(\omega)\right]$ entering in Eq. (4.15) after performing the matching to the near zone (see Appendix B). These terms, which can be written in compact form as

$$
\begin{aligned}
\mathcal{L}_{4 \mathrm{PN}, \Delta \mathrm{S}_{\text {rad }} \text { (finite) }}^{\text {tail (far) }}= & -4\left[\frac{G^{4} m_{1}^{3} m_{2}}{r^{4}}\left(5 \boldsymbol{v}^{2}-4 \frac{(\boldsymbol{v} \cdot \boldsymbol{r})^{2}}{r^{2}}\right)\right. \\
& \left.+\frac{G^{5} m_{1}^{3} m_{2}^{2} M_{0}}{r^{5}}\right]+(1 \leftrightarrow 2)+\cdots,
\end{aligned}
$$

must be kept in the renormalized Lagrangian [in addition to the extra ones in the third line of Eq. (4.10)] to arrive at correct (and unambiguous) physical expressions. ${ }^{18}$ The ellipses in the above equation account for other (finite) contributions which are zero in lower-order equations of motion, and are therefore irrelevant for all physical purposes.

\footnotetext{
${ }^{18}$ In the Fokker-action approach, using a (short-distance) worldline redefinition to remove the (long-distance) IR poles [26], the "extra terms" are due to $\mathcal{O}(\epsilon)$ corrections to the equations of motion in $d$ dimensions. Our approach, on the other hand, illustrates the true origin of these terms.
}

\section{EFFECTIVE THEORY TO 4PN ORDER}

The renormalization procedure described in this paper,

$$
\begin{aligned}
& \left(\mathcal{L}_{n \mathrm{PN}}^{\mathrm{UV}(\text { near+self })}+\mathcal{L}_{n \mathrm{PN}}^{\text {c.t. (near) }}\right)+\left(\mathcal{L}_{n \mathrm{PN}}^{\mathrm{UV}(\mathrm{IR} \text { near+self-ZB })}+\mathcal{L}_{n \mathrm{PN}}^{\mathrm{UV}(\mathrm{far})}\right) \\
& \quad \rightarrow \text { finite, }
\end{aligned}
$$

explains how the intermediate IR and UV divergences are removed, or cancel out, from the renormalized effective action at a given $n \mathrm{PN}$ order. At the end of the day, including all of the finite pieces, we are left with a series of local- and nonlocal-in-time contributions to the Lagrangian, which we will display momentarily. We also have a series of logarithmic terms, of which only the (long-distance) one shown in Eq. (2.10) contributes to physical quantities.

\section{A. Long-distance logarithms}

In addition to the poles, we have also demonstrated how the factors of $\log \mu$ (associated with IR divergences in the potential region) cancel out against conservative UV logarithms from the tail integrals in the far zone. This is not surprising, and it is entirely due to the general rule

$$
G_{d}^{n} / \epsilon \equiv\left(\mu^{-\epsilon} G\right)^{n} / \epsilon \rightarrow G^{n}(1 / \epsilon-n \log \mu)
$$

which links the poles to the factors of $\log \mu$ in $\operatorname{dim}$. reg. at a given $n$th order in $G$.

As we argued, while the $\log \mu$ 's disappear, long-distance logarithms of the form $\log \omega r$ remain in the Lagrangian. such as the dipole term at 3PN [see Eq. (4.9)]. This correction in particular is proportional to a term which 
vanishes on shell, and therefore it does not contribute to physical quantities. However, for the case of the quadrupole term in Eq. (2.10) at 4PN order, the key difference is that the associated $\log \omega r$ is no longer proportional to a term which vanishes on shell, due to a conservation law. For all of the other corrections induced by Eq. (4.13), which vanish upon using moment relations, we can show that there is no observable contribution provided all of the relevant terms are included [see Eq. (4.20)].

\section{B. Short-distance logarithms}

There are also factors of $\log \bar{\mu} r$ associated to UV divergences in the potential region. They are also due to Eq. (5.2), and the expansion of the $d$-dimensional Green's (as well as $\Gamma$ ) functions around $d=3$ in dim. reg. These logarithms remain after removing the UV poles through counterterms [e.g., Eq. (3.15)], while the finite pieces are renormalization-scheme dependent. The $\mu$ dependence, on the other hand, is absorbed into renormalized coefficients $c_{\alpha, \text { ren }}(\mu)$, such that we have a renormalization group equation

$$
\mu \frac{d}{d \mu} c_{\alpha, \text { ren }}(\mu)=\beta_{\alpha} G^{n} m^{n+1}
$$

following from the condition $\mu \frac{d}{d \mu} \mathcal{L}=0$, and the general structure

$$
\begin{aligned}
\mathcal{L}= & \cdots+\left(c_{\alpha, \text { ren }}^{(a)}(\mu)-\beta_{\alpha} G^{n} m_{a}^{n+1} \log \mu r+\cdots\right) \\
& \times f_{\alpha}\left(\boldsymbol{x}_{a}, \boldsymbol{v}_{a}, \boldsymbol{b}_{a}, \cdots\right)+\cdots
\end{aligned}
$$

of the renormalized Lagrangian. In general, these types of logarithms contribute to physical quantities, and the renormalization group equation allows us to resum many of such contributions (e.g., Refs. [17,43]). However, for the case at hand the fact that the $c_{\alpha, \text { ren }}^{(a)}(\mu)$ coefficients can be removed by field redefinitions implies that, likewise, the logarithmic running does not contribute to physical quantities. [In other words, $f_{\alpha}\left(\boldsymbol{x}_{a}, \boldsymbol{v}_{a}, \boldsymbol{b}_{a}, \cdots\right)$ vanishes on shell to 4PN order.]

To illustrate the situation, let us consider once again the 3PN case. After implementing the MS scheme, we have ${ }^{19}$

\footnotetext{
${ }^{19} \mathrm{We}$ could have equally used the $\overline{\mathrm{MS}}$ scheme, and subtracted the UV poles plus all of the constants going into the definition of $\bar{\mu}$ in Eq. (2.5). The difference is an inconsequential shift in the renormalized parameters.
}

$$
\begin{aligned}
\mathcal{L}_{3 \mathrm{PN}}^{\mathrm{UV}(\text { near }+ \text { self })}+\mathcal{L}_{3 \mathrm{PN}}^{\left.c_{a i}, \text { (near }\right)} & \\
= & \left(c_{a \dot{v}, \text { ren }}^{(1)}(\mu)-\frac{22}{3} G^{2} m_{1}^{3} \log \bar{\mu} r+\cdots\right) \boldsymbol{a}_{1} \\
& \cdot\left(\boldsymbol{a}_{1}+\frac{G m_{2} \boldsymbol{r}}{r^{3}}\right)+(1 \leftrightarrow 2),
\end{aligned}
$$

after adding Eqs. (3.7) and (3.14) and splitting the bare coefficient $c_{a i}^{(1)}$ into a counterterm [given in Eq. (3.15)] plus $c_{a \dot{v} \text {,ren }}^{(1)}(\mu)$ (the renormalized piece). ${ }^{20}$ It is now straightforward to show that the choice ${ }^{21}$

$$
\mu=(r \sqrt{4 \pi})^{-1} e^{-\gamma_{E} / 2}
$$

removes the logarithmic contribution, as well as some associated constants. The logarithmic contributions are then encoded in the running of the renormalized coefficient. Yet, the entire term multiplies a factor that is proportional to the leading-order equation of motion, and therefore it can be removed from physical quantities. In our language, the $c_{a i}$ coefficient can be set to zero by a field redefinition, thus erasing all information about factors of $\log \bar{\mu} r$.

\section{Renormalized Lagrangian}

Below we quote the final expression for the renormalized Lagrangian to $4 \mathrm{PN}$ order, using the intermediate results reported in Refs. [14-18], together with the procedure described in Eq. (5.1) $[19,20]$. The reader will find no trace of $\log \mu(\operatorname{or} \log \bar{\mu})$, either because of the cancellation between near-/far-zone contributions we discussed above, or because they are absorbed into coefficients which can be removed by field redefinitions. The leftover factors of $\log \omega r$ from the radiation region are also omitted, except for the surviving term shown in Eq. (4.10). This is the only contribution which is not proportional to a quantity that vanishes on shell.

After all is said and done, the resulting effective Lagrangian can be written as

$$
\mathcal{L}_{3,4 \mathrm{PN}}=\mathcal{L}_{0123 \mathrm{PN}}+\mathcal{L}_{4 \mathrm{PN}}
$$

$\mathcal{L}_{0123 \mathrm{PN}}$ is the finite part of the Lagrangian in harmonic gauge to 3PN order, as shown in Ref. [14] (see also Ref. [46]), except for the $G^{3}$ and $G^{4}$ contributions, which for us here should read

\footnotetext{
${ }^{20}$ Since the counterterm lives in the (one-dimensional) worldline, there are no factors of $\mu$ associated with $c_{a i}$ in $d$ bulk dimensions. Notice, however, that the logarithmic term in Eq. (3.14) (due to the $d$-dimensional Green's function) is essential for obtaining the form in Eq. (5.5). The same applies for the logarithmic contributions in Eq. (3.16) at 4PN order.

${ }^{21}$ Formally speaking, the variation of the action must be performed prior to choosing the value of $\mu$. However, only the variation of $f_{\alpha}(\cdots)$ contributes. Therefore, setting $\mu$ in the action is de facto innocuous.
} 


$$
\begin{aligned}
\mathcal{L}_{3 \mathrm{PN}}^{G^{3,4}}= & \frac{G^{3} m_{1}^{3} m_{2}}{r^{3}}\left[\frac{209}{18} \boldsymbol{v}_{1}^{2}-\frac{118}{9} \boldsymbol{v}_{1} \cdot \boldsymbol{v}_{2}+\frac{5}{4} \boldsymbol{v}_{2}^{2}-\frac{355}{12}\left(\boldsymbol{v}_{1} \cdot \boldsymbol{n}\right)^{2}+\frac{82}{3}\left(\boldsymbol{v}_{1} \cdot \boldsymbol{n}\right)\left(\boldsymbol{v}_{2} \cdot \boldsymbol{n}\right)+\frac{3}{2}\left(\boldsymbol{v}_{2} \cdot \boldsymbol{n}\right)^{2}\right] \\
& +\frac{G^{3} m_{1}^{2} m_{2}^{2}}{r^{3}}\left[-\frac{305}{72} \boldsymbol{v}_{1}^{2}+\frac{439}{144} \boldsymbol{v}_{1} \cdot \boldsymbol{v}_{2}+\frac{383}{24}\left(\boldsymbol{v}_{1} \cdot \boldsymbol{n}\right)^{2}-\frac{889}{48}\left(\boldsymbol{v}_{1} \cdot \boldsymbol{n}\right)\left(\boldsymbol{v}_{2} \cdot \boldsymbol{n}\right)\right. \\
& \left.+\frac{41 \pi^{2}}{64}\left(\boldsymbol{v} \cdot \boldsymbol{v}_{1}-3(\boldsymbol{v} \cdot \boldsymbol{n})\left(\boldsymbol{v}_{1} \cdot \boldsymbol{n}\right)\right)\right]-\frac{3}{8} \frac{G^{4} m_{1}^{4} m_{2}}{r^{4}}-\frac{67}{3} \frac{G^{4} m_{1}^{3} m_{2}^{2}}{r^{4}}+(1 \leftrightarrow 2),
\end{aligned}
$$

where we introduced $\boldsymbol{n} \equiv \boldsymbol{r} / \boldsymbol{r}$. The modified version of the 3PN Lagrangian is due to undoing integration by parts in the terms shown in Ref. [14], which was required here to properly identify the coefficients of the logarithms in the near zone. ${ }^{22}$

The 4PN effective action can be written as follows:

$$
\mathcal{L}_{4 \mathrm{PN}}=\frac{7}{256} m_{1} v_{1}^{10}+\mathcal{L}_{4 \mathrm{PN}}^{G}+\mathcal{L}_{4 \mathrm{PN}}^{G^{2}}+\mathcal{L}_{4 \mathrm{PN}}^{G^{3}}+\mathcal{L}_{4 \mathrm{PN}}^{G^{4}}+\mathcal{L}_{4 \mathrm{PN}}^{G^{5}}+\mathcal{L}_{4 \mathrm{PN}}^{G^{2} \log v}
$$

where the last term, introduced in Eq. (4.12), encodes the nonlocal-in-time contribution from the far zone due to the tail effect. We have absorbed the celebrated $\frac{41}{30}$ in Eq. (2.10) into the local part of the effective action, and subsequently reduced it using the double-zero trick, turning it into an $\mathcal{O}\left(G^{4}\right)$ contribution. This means that, in practice, the first two local terms from the near zone, $\mathcal{L}_{4 \mathrm{PN}}^{G}+\mathcal{L}_{4 \mathrm{PN}}^{G^{2}}$, remain as reported in Ref. [15] [see Eqs. (13) and (26)], while the others take the form

$$
\begin{aligned}
& \mathcal{L}_{4 \mathrm{PN}}^{G^{3}}=\frac{G^{3} m_{1}^{3} m_{2}}{r^{3}}\left[\boldsymbol{a}_{1} \cdot \boldsymbol{v}_{2}\left(\frac{3763}{240} \boldsymbol{v}_{2} \cdot \boldsymbol{r}-\frac{18719}{720} \boldsymbol{v}_{1} \cdot \boldsymbol{r}\right)+\boldsymbol{a}_{1} \cdot \boldsymbol{r}\left(-\frac{18719}{1440} \boldsymbol{v}_{1}^{2}-\frac{95119}{7200} \boldsymbol{v}^{2}+\frac{1309}{48}\left(\boldsymbol{v}_{2} \cdot \boldsymbol{n}\right)^{2}-\frac{75}{4}\left(\boldsymbol{v}_{1} \cdot \boldsymbol{n}\right)\left(\boldsymbol{v}_{2} \cdot \boldsymbol{n}\right)\right)\right. \\
& +\frac{3763}{480}\left(\boldsymbol{a}_{2} \cdot \boldsymbol{r}\right) \boldsymbol{v}_{1}^{2}-\frac{231}{160} \boldsymbol{v}_{1}^{4}+\frac{1397}{480} \boldsymbol{v}_{1}^{2} \boldsymbol{v}_{2}^{2}-\frac{433}{60} \boldsymbol{v}_{1}^{2}\left(\boldsymbol{v}_{1} \cdot \boldsymbol{v}_{2}\right)+\frac{43}{2}\left(\boldsymbol{v}_{1} \cdot \boldsymbol{v}_{2}\right)\left(\boldsymbol{v} \cdot \boldsymbol{v}_{2}\right)+\frac{91}{16} \boldsymbol{v}_{2}^{4} \\
& +\boldsymbol{v}_{1}^{2}\left(\frac{3463}{160}\left(\boldsymbol{v}_{1} \cdot \boldsymbol{n}\right)^{2}-\frac{1047}{20}\left(\boldsymbol{v}_{1} \cdot \boldsymbol{n}\right)\left(\boldsymbol{v}_{2} \cdot \boldsymbol{n}\right)+\frac{3923}{160}\left(\boldsymbol{v}_{2} \cdot \boldsymbol{n}\right)^{2}\right)+\boldsymbol{v}_{1} \cdot \boldsymbol{v}_{2}\left(7\left(\boldsymbol{v}_{1} \cdot \boldsymbol{n}\right)\left(\boldsymbol{v}_{2} \cdot \boldsymbol{n}\right)+\frac{43}{16}\left(\boldsymbol{v}_{1} \cdot \boldsymbol{n}\right)^{2}-2\left(\boldsymbol{v}_{2} \cdot \boldsymbol{n}\right)^{2}\right) \\
& \left.+\boldsymbol{v}_{2}^{2}\left(\frac{7}{4}\left(\boldsymbol{v}_{2} \cdot \boldsymbol{n}\right)^{2}-\frac{1}{8}\left(\boldsymbol{v}_{1} \cdot \boldsymbol{n}\right)^{2}-\frac{7}{2}\left(\boldsymbol{v}_{1} \cdot \boldsymbol{n}\right)\left(\boldsymbol{v}_{2} \cdot \boldsymbol{n}\right)\right)+\left(\boldsymbol{v}_{1} \cdot \boldsymbol{n}\right)^{2}\left(\frac{5}{2}\left(\boldsymbol{v}_{1} \cdot \boldsymbol{n}\right)^{2}+\frac{35}{16}\left(\boldsymbol{v}_{1} \cdot \boldsymbol{n}\right)\left(\boldsymbol{v}_{2} \cdot \boldsymbol{n}\right)-\frac{15}{4}\left(\boldsymbol{v}_{2} \cdot \boldsymbol{n}\right)^{2}\right)\right] \\
& +\frac{G^{3} m_{1}^{2} m_{2}^{2}}{r^{3}}\left[\boldsymbol{a} \cdot \boldsymbol{r}\left(\left(\frac{349207}{7200}-\frac{43}{128} \pi^{2}\right) \boldsymbol{v}^{2}+\left(\frac{123 \pi^{2}}{128}-\frac{2005}{96}\right)\left(\boldsymbol{v}_{2} \cdot \boldsymbol{n}\right)^{2}\right)\right. \\
& +\left(\left(\boldsymbol{a}_{1} \cdot \boldsymbol{r}\right) \boldsymbol{v}_{1}^{2}+2\left(\boldsymbol{v}_{1} \cdot \boldsymbol{n}\right)\left(\boldsymbol{v}_{2} \cdot \boldsymbol{a}_{1}\right)\right)\left(\frac{1099}{288}-\frac{41 \pi^{2}}{128}\right)+\frac{383}{192} \boldsymbol{v}_{1}^{4}+\left(\frac{21427}{480}+\frac{133 \pi^{2}}{1024}\right)\left(\boldsymbol{v}_{1}^{2} \boldsymbol{v}^{2}-2\left(\boldsymbol{v}_{1} \cdot \boldsymbol{v}_{2}\right)\left(\boldsymbol{v} \cdot \boldsymbol{v}_{1}\right)\right)-\frac{55}{24} \boldsymbol{v}_{1}^{2}\left(\boldsymbol{v}_{1} \cdot \boldsymbol{v}_{2}\right) \\
& +\boldsymbol{v}_{1} \cdot \boldsymbol{v}_{2}\left(\frac{32887}{150}\left(\boldsymbol{v}_{1} \cdot \boldsymbol{n}\right)^{2}-\frac{33487}{150}\left(\boldsymbol{v}_{1} \cdot \boldsymbol{n}\right)\left(\boldsymbol{v}_{2} \cdot \boldsymbol{n}\right)-\frac{447 \pi^{2}}{256}(\boldsymbol{v} \cdot \boldsymbol{n})\left(\boldsymbol{v}_{1} \cdot \boldsymbol{n}\right)\right) \\
& +\boldsymbol{v}_{1}^{2}\left(\frac{270521}{1200}\left(\boldsymbol{v}_{1} \cdot \boldsymbol{n}\right)\left(\boldsymbol{v}_{2} \cdot \boldsymbol{n}\right)-\frac{275321}{2400}\left(\boldsymbol{v}_{1} \cdot \boldsymbol{n}\right)^{2}-\frac{64799}{600}\left(\boldsymbol{v}_{2} \cdot \boldsymbol{n}\right)^{2}+\frac{447 \pi^{2}}{512}(\boldsymbol{v} \cdot \boldsymbol{n})^{2}\right) \\
& +\left(\boldsymbol{v}_{1} \cdot \boldsymbol{n}\right)^{2}\left(\frac{155947}{2880}\left(\boldsymbol{v}_{1} \cdot \boldsymbol{n}\right)^{2}-\frac{155977}{720}\left(\boldsymbol{v}_{1} \cdot \boldsymbol{n}\right)\left(\boldsymbol{v}_{2} \cdot \boldsymbol{n}\right)+\frac{78911}{480}\left(\boldsymbol{v}_{2} \cdot \boldsymbol{n}\right)^{2}\right. \\
& \left.\left.-\frac{2155 \pi^{2}}{1024}\left(\left(\boldsymbol{v}_{1} \cdot \boldsymbol{n}\right)^{2}-4\left(\boldsymbol{v}_{1} \cdot \boldsymbol{n}\right)\left(\boldsymbol{v}_{2} \cdot \boldsymbol{n}\right)+3\left(\boldsymbol{v}_{2} \cdot \boldsymbol{n}\right)^{2}\right)\right)\right]+(1 \leftrightarrow 2),
\end{aligned}
$$

\footnotetext{
${ }^{22}$ More concretely, the difference is in the term $\frac{1}{r^{3}}\left(\boldsymbol{r} \cdot \boldsymbol{a}_{1}\right) \log \mu \boldsymbol{r}$ we used earlier, instead of the expression $\frac{1}{r^{3}}\left(\boldsymbol{v} \cdot \boldsymbol{v}_{1}-\frac{3}{r^{2}}(\boldsymbol{r} \cdot \boldsymbol{v})\left(\boldsymbol{r} \cdot \boldsymbol{v}_{1}\right)\right) \log \mu r$, which appears in the literature.
} 


$$
\begin{aligned}
\mathcal{L}_{4 \mathrm{PN}}^{G^{4}}= & \frac{G^{4} m_{1}^{4} m_{2}}{r^{4}}\left[-\frac{98549}{3600} \boldsymbol{v}_{1}^{2}+\frac{95849}{3600} \boldsymbol{v}_{1} \cdot \boldsymbol{v}_{2}+\frac{15}{16} \boldsymbol{v}_{2}^{2}+\frac{103949}{900}\left(\boldsymbol{v}_{1} \cdot \boldsymbol{n}\right)^{2}-\frac{105299}{900}\left(\boldsymbol{v}_{1} \cdot \boldsymbol{n}\right)\left(\boldsymbol{v}_{2} \cdot \boldsymbol{n}\right)+\frac{9}{4}\left(\boldsymbol{v}_{2} \cdot \boldsymbol{n}\right)^{2}\right] \\
& +\frac{G^{4} m_{1}^{3} m_{2}^{2}}{r^{4}}\left[-\left(\frac{104569}{7200}+\frac{15}{32} \pi^{2}\right) \boldsymbol{v}_{1}^{2}+\left(\frac{103}{16} \pi^{2}-\frac{11923}{240}\right) \boldsymbol{v}_{1} \cdot \boldsymbol{v}_{2}+\left(\frac{542659}{7200}-\frac{191}{32} \pi^{2}\right) \boldsymbol{v}_{2}^{2}\right. \\
& \left.+\left(\frac{659}{96} \pi^{2}-\frac{125209}{7200}\right)\left(\boldsymbol{v}_{1} \cdot \boldsymbol{n}\right)^{2}+\left(\frac{296893}{720}-\frac{1715}{48} \pi^{2}\right)\left(\boldsymbol{v}_{1} \cdot \boldsymbol{n}\right)\left(\boldsymbol{v}_{2} \cdot \boldsymbol{n}\right)+\left(\frac{2771}{96} \pi^{2}-\frac{871207}{2400}\right)\left(\boldsymbol{v}_{2} \cdot \boldsymbol{n}\right)^{2}\right] \\
& +(1 \leftrightarrow 2),
\end{aligned}
$$

$$
\mathcal{L}_{4 \mathrm{PN}}^{G^{5}}=\frac{3}{8} \frac{G^{5} m_{1}^{5} m_{2}}{r^{5}}+\frac{G^{5} m_{1}^{4} m_{2}^{2}}{r^{5}}\left(\frac{94931}{3600}+\frac{105}{32} \pi^{2}\right)+\frac{G^{5} m_{1}^{3} m_{2}^{3}}{r^{5}}\left(\frac{225839}{2400}-\frac{71}{32} \pi^{2}\right)+(1 \leftrightarrow 2) .
$$

Our final result can be shown to be equivalent to the one obtained in Refs. [23,29] leading, for instance, to the same expression for the binding energy and periastron advance.

\section{Lorentz invariance}

There is only one minor caveat in the steps leading to the above expression for the renormalized Lagrangian. Prior to removing the UV $\log \bar{\mu} r$ 's, the effective theory is Lorentz invariant to 4PN order (up to double zeros), with $\mu$ treated as a constant. Since $r=\left|x_{1}-x_{2}\right|$ transforms under a boost [62], the choice $\bar{\mu}=1 / r$, which removes the logarithms, has the result of making the action Lorentz invariant only on shell. Of course, this is inconsequential, as any choice of $\mu$ is physically equivalent. Nonetheless, a simple way to recover manifest Lorentz invariance of the effective action is by means of a worldline redefinition. In particular, it is easy to see that the transformation $\delta \boldsymbol{x}_{a}(t)=-11 G^{2} m_{a}^{2}\left(\boldsymbol{v}_{b} \cdot \boldsymbol{r}\right)^{2} /\left(3 r^{2}\right) \boldsymbol{a}_{a}$ introduces an extra term,

$\Delta \mathcal{L}_{\mathrm{LI}}=\frac{11 G^{2} m_{1}^{3}}{3} \frac{\left(\boldsymbol{v}_{2} \cdot \boldsymbol{r}\right)^{2}}{r^{2}}\left(\boldsymbol{a}_{1}+\frac{G m_{2} \boldsymbol{r}}{r^{3}}\right) \cdot \boldsymbol{a}_{1}+(1 \leftrightarrow 2)$,

which readily reinstates manifest (perturbative) Lorentz invariance. Notice, as expected, that it vanishes on shell. While it is straightforward to introduce this or other similar terms, we did not include it since it does not affect observable quantities.

\section{DISCUSSION}

The existence of intermediate IR/UV divergences, as well as the explicit cancellation, is entirely a byproduct of the split into near and far zones, the asymptotic expansion of Feynman integrals [63] and the use of the method of regions with potential and radiation modes (e.g., Refs. [64,65]). In principle, we do not need to perform this splitting, since we can always work with a point-particle effective theory and relativistic propagators, performing instead the post-Minkowskian (PM) expansion. ${ }^{23}$ It is only for convenience, and to separate the relevant physics in the near and far regions, that potential and radiation modes are introduced. The price to pay, however, for the simplification of the Feynman integrals is the introduction of new divergences which are not present in the original theory. In our case, spurious IR/UV singularities appear from the near/far expansions of the iterated Green's functions in the PN formalism, already at $\mathcal{O}\left(G^{2}\right)$ (i.e., "one loop").

For example, let us return to the topology in Fig. 2, and consider the full propagators without expanding in the regions of the nonrelativistic limit. Concentrating on the IR properties, it is easy to see that the diagram is dominated by a triangle integral,

$\Delta\left(p, \sigma_{1}\right)=\int d \sigma_{1}^{\prime} \int \frac{d^{D} k}{(2 \pi)^{D}} \frac{1}{k^{2}(p-k)^{2}} e^{i k_{\mu}\left(x_{1}^{\mu}\left(\sigma_{1}\right)-x_{1}^{\mu}\left(\sigma_{1}^{\prime}\right)\right)}$,

in $D$ dimensions. Notice that for $D=4$ this integral is both UV and IR finite for bound states. The remaining integral consists of a Fourier transform. The exact form depends on the numerator, which carries extra powers of momenta. Schematically, it takes the form

$\int d \sigma_{1} d \sigma_{2} \int \frac{d^{D} p}{(2 \pi)^{D}} \Delta\left(p, \sigma_{1}\right) \frac{p^{\alpha} p^{\beta}}{p^{2}} e^{i p_{\mu}\left(x_{1}^{\mu}\left(\sigma_{1}\right)-x_{2}^{\mu}\left(\sigma_{2}\right)\right)}$.

In the static limit, the triangle in Eq. (6.1) collapses into a $d=(D-1)$ integral, such that we end up with a threedimensional Fourier transform to obtain the $G^{2}$ correction to the potential at first $\mathrm{PN}$ order, proportional to

\footnotetext{
${ }^{23}$ The computation of the classical limit of scattering amplitudes in the PM framework has recently received renewed attention, due to their ability to extract the conservative Hamiltonian of the two-body problem at a given PM order through a matching calculation [66-68] (see also Refs. [69-80] and references therein).
} 
$\int d^{3} \boldsymbol{p}|\boldsymbol{p}|^{-1} e^{i \boldsymbol{p} \cdot \boldsymbol{r}} \propto 1 / r^{2}$ in $d=3$, as expected. For the general case the integral is more complicated; however, the result is IR finite by power counting.

The situation changes when we use the method of regions $[64,65]$. Expanding the propagators with potential modes in powers of $p_{0} /|\boldsymbol{p}|$ inside the integrals, it is easy to see that one of the terms is of the form

$$
\boldsymbol{a}_{1}^{i} \boldsymbol{a}_{2}^{j} \int d t \int \frac{d^{3} \boldsymbol{p}}{(2 \pi)^{3}} \frac{\boldsymbol{p}^{i} \boldsymbol{p}^{j}}{|\boldsymbol{p}|^{5}} e^{-i \boldsymbol{p} \cdot\left(\boldsymbol{x}_{1}(t)-\boldsymbol{x}_{2}(t)\right)},
$$

which is logarithmically IR divergent. The key difference is that, for quasi-instantaneous interactions, the $p_{0}$ integrals are traded for time derivatives on the worldlines, which in the classical EFT are treated as external (nonpropagating) sources. At the same time, the IR properties of the diagram change due to the $1 /|\boldsymbol{p}|$ factors. These IR divergences, which do not belong to the near zone, are therefore clearly an artifact of the expansion into regions. It comes as no surprise then that they will be linked to (and cancel against) singularities associated with radiation modes.

For the conservative contribution from the far zone, we must consider on-shell modes with $p_{0} \simeq|\boldsymbol{p}| \simeq$ $1 / \lambda_{\text {rad }}$, where $\lambda_{\text {rad }} \sim r / v$, as well as long-distance quasiinstantaneous (potential) modes with $p_{0} \ll|\boldsymbol{p}| \sim 1 / \lambda_{\text {rad }}$. The latter build up the (Kerr) background geometry produced by the binary as a whole in the far zone. The propagators for the (off-shell) potential modes are expanded as in the near region, but not the (on-shell) radiation modes. In the nonrelativistic limit, we have $\boldsymbol{p} \cdot \boldsymbol{r} \simeq r / \lambda_{\text {rad }} \ll 1$ and therefore the (spatial part of the) exponential in Eq. (6.2) is expanded instead. Hence, rather than having a one-loop integral plus a Fourier transform for the topology in Fig. 2, with radiation modes we end up with a two-loop-type integral. In the EFT approach this is represented by a diagram with the topology of Fig. 3, with the binary described as a localized source endowed with a series of multipole moments. ${ }^{24}$ For the case at hand in Eq. (6.2), after expanding in the radiation region, we find the first the term

$$
\int \frac{d \omega}{\pi} \int \frac{d^{3} \boldsymbol{p}}{(2 \pi)^{3}} \frac{d^{3} \boldsymbol{k}}{(2 \pi)^{3}} \boldsymbol{x}_{1}^{i}(\omega) \boldsymbol{x}_{1}^{j}(\omega) \frac{\boldsymbol{k}^{i} \boldsymbol{k}^{j}}{\boldsymbol{p}^{2} k^{2}(p+k)^{2}},
$$

\footnotetext{
${ }^{24}$ For more complicated topologies, as in Fig. 1, the contribution from radiation modes is also described by a diagram similar to Fig. 3, but with the source multipoles incorporating higher-order corrections in $G$. This is handled in the EFT approach in two steps: first, by matching the source multipoles in the long-distance EFT to the worldline effective theory (integrating out the potential modes for the two bodies), and second, by computing the (two-loop) tail diagram. See Appendix B for more details.
}

with $k^{2} \equiv \omega^{2}-\boldsymbol{k}^{2}+i \epsilon$ and $(p+k)^{2}=\omega^{2}-(\boldsymbol{q}+\boldsymbol{k})^{2}+i \epsilon$. This (two-loop) integral is UV divergent, and proportional to derivatives of the dipole moment associated with the binary. Adding extra terms in the expansion of the exponential, it is straightforward to show that the far-zone contribution is UV divergent and can be written in terms of derivatives of the source multipoles. As for the IR poles from the potential region, the UV divergence is likewise artificial, and it is due to performing a multipole expansion. As anticipated, after the subtraction of the zero bin, by construction the poles cancel out against the equally unphysical divergence from the near region $[19,20]$. Because the binary is shrunk to a point, some of the divergences introduced by the multipole expansion will match into self-energy terms in the potential region. This is the reason additional scaleless integrals are needed to make manifest the cancellation. In the above case, the dipole term does not contribute to physical quantities. However, starting with the (trace-free) quadrupole, radiation modes contribute to the conservative sector, as explained here.

Needless to say, as a systematic approximation to the full answer the PN expansion of the relativistic expression must be captured by the decomposition into potential and radiation modes (albeit introducing intermediate spurious poles). Yet, to correctly perform the expansion into regions, and in particular when different regions overlap as it happens in our case, we had to implement the zero-bin subtraction [33]. In this procedure, in which the IR sensitivity in the near zone is removed from the region of integration, there are leftover UV poles. The latter cancel out against the UV poles produced by the multipole-expanded computation involving radiation modes. Had we not expanded the integral(s) into regions, these spurious divergences would not appear. Yet, their existence is not a mystery in the EFT framework. The method of regions is a useful tool to isolate the relevant physics one scale at a time, but the procedure may introduce artificial divergences which must be properly removed $[19,20]{ }^{25}$

In conclusion, in this paper we have derived the renormalized effective action describing the dynamics of binary systems to 4PN order. We have shown how to implement dim. reg. when IR and UV divergences appear in intermediate steps, paying special attention to the contribution from scaleless integrals. We have renormalized away the near-zone UV poles by the use of counterterms in the point-particle effective theory. As expected from the effacement theorem [81], the counterterms can be removed by field redefinitions at this order

\footnotetext{
${ }^{25}$ As a perk of using the method of regions, the appearance of UV poles in the long-distance theory allows us to resum (universal) logarithmic corrections using the renormalization group $[17,19,20]$.
} 
(e.g., Ref. [8]). ${ }^{26}$ We have also shown how the spurious IR divergences in the potential region are handled by means of the zero-bin subtraction [33], which unambiguously removes from the Feynman diagrams the region of integration which does not belong to the near zone $[19,20]$. The leftover UV poles which result from this procedure cancel out against equally unphysical divergences in radiationreaction effects.

Our derivation of the 4PN renormalized Lagrangian differs from the methodologies used in Refs. [21-29], mainly for two reasons. First of all, we have implemented a regularization-independent procedure to remove IR divergences which uniquely determines the gravitational potential, thus not requiring the introduction of ambiguity parameters nor information outside of the realm of the PN expansion. Second, contrary to what is ultimately the case in the Fokker-action approach [29], we did not remove near-zone IR poles combined with UV divergences in tail terms by means of redefinitions of the particles' worldlines. ${ }^{27}$ Instead, we have identified the IR/UV singularities from the different regions and demonstrated the explicit cancellation of the spurious near-/far-zone divergences after the subtraction of the zero bin. While the procedure is independent of the regulator, we have performed all of the calculations within the confines of dim. reg., as it is customary in an EFT approach. This is in contrast to the results in Refs. [21-29] where an additional regulator appears to be a necessity. Hence, in comparison with the Fokker-action [6] and ADM [7] approaches, in our opinion the EFT formalism provides a more systematic derivation of the conservative dynamics, which furthermore can be naturally extended to all PN orders without ambiguity parameters or extra regulators.

We have demonstrated the ability of the EFT approach to tackle intricate calculations in a systematic and scalable

\footnotetext{
${ }^{26}$ Let us stress an important point. In order to remove the $c_{a i}$ coefficient by a field redefinition, spin and finite-size effects must be ignored. Once spin is included, the field redefinition $\delta x^{\mu}=$ $-c_{a \dot{v}} \dot{v}^{\mu}$ leads to$$
-c_{a \dot{v}}\left(a_{\mu}-\frac{1}{2 m} R_{\mu \nu \alpha \beta} v^{\nu} S^{\alpha \beta}+\cdots\right) i^{\mu},
$$

with the ellipses including terms quadratic in the spin Ref. [12]. Hence, the structure of UV poles must be such that they are canceled by the above operator (plausibly including other spindependent counterterms), with the precise coefficient given in Eq. (3.15). This provides a nontrivial consistency check for our procedure.

${ }^{27}$ Let us emphasize that the lack of a systematic treatment of IR/UV divergences can potentially lead to inconsistencies at higher PN orders, in particular when UV divergences in the potential region (and related length scales and logarithms) are associated with physical finite-size effects. Unlike worldline redefinitions, counterterms and renormalized parameters in the effective theory can still be applied in such a case, with operators that do not vanish on shell.
}

fashion within the PN framework. Yet, the future enterprise in precision GW physics is vast, with the associated complexity increasing at every iteration. In order to move forward, reaching the physically motivated threshold at 5PN order (and beyond) where the first finite-size operators appear in Eq. (2.1) [3,4] may require significant advances both in our understanding of the theoretical foundations and computational efficiency (see Ref. [31] for partial results at 5PN order in the EFT approach, as well as Ref. [32]). In a parallel development, the study of scattering amplitudes and other ideas from particle physics has opened up new routes to simplify calculations for the conservative sector, and plausibly also for the radiated power (e.g., Refs. [66-80]). In principle, these new tools can further streamline the relevant PN computations within the EFT approach, which we find is a natural venue for future explorations.

\section{ACKNOWLEDGMENTS}

We thank Luc Blanchet, Thibault Damour, Guillaume Faye, Adam Leibovich, and Gerhard Schäfer for very useful discussions and comments. We would like to thank ICTPSAIFR as well as the Mainz Institute for Theoretical Physics, for the support to organize the workshops "Analytic Methods in General Relativity" 28 and "The Sound of Spacetime: The Dawn of Gravitational Wave Science," ${ }^{29}$ respectively, where this work originated and preliminary results were presented. We thank Nordita and the organizers of the workshop "QCD Meets Gravity IV",30 for hospitality while this work was being completed. S. F. is supported by the Fonds National Suisse and by the SwissMap NCCR. R. A. P. acknowledges financial support from the ERC Consolidator Grant "Precision Gravity: From the LHC to LISA" provided by the European Research Council (ERC) under the European Union's H2020 research and innovation programme (Grant Agreement No. 817791), as well as from the Deutsche Forschungsgemeinschaft (DFG, German Research Foundation) under Germany's Excellence Strategy (EXC 2121) "Quantum Universe" (390833306). R. A. P. would also like to thank the Simons Foundation and FAPESP (Young Investigator Awards) for support during the early stages of this work, and the organizers and participants of the "Simons Foundation Symposium: Amplitudes meet Cosmology" ${ }^{31}$ for the opportunity to present this work and for helpful discussions. R. S. has been supported for part of the duration of the present work by the FAPESP Grant No. 2012/14132-3, and wishes to thank the Physics Department at the University of Geneva for hospitality and support during his visits. R.S. acknowledges the High-Performance Computing Center at UFRN.

\footnotetext{
${ }^{28} \mathrm{http}: / /$ www.ictp-saifr.org/gr2016.

${ }^{29} \mathrm{https}$ ///indico.mitp.uni-mainz.de/event/124/.

${ }^{30} \mathrm{http}: / /$ www.nordita.org/qcd2018.

${ }^{31} \mathrm{https}$ ://www.simonsfoundation.org/event/amplitudes-meetcosmology-2019/.
} 


\section{APPENDIX A: FEYNMAN RULES AND MASTER INTEGRALS}

To compute the contribution from the near zone it is convenient to decompose the metric field $\left(g_{\mu \nu}\right)$ in terms of scalar, vector, and tensor perturbations: $\left(\phi, \boldsymbol{A}, \gamma_{i j}\right)$. The gauge-fixed Einstein-Hilbert action in $(d+1)$ dimensions,

$$
S_{\mathrm{EH}}=-\frac{1}{16 \pi G_{d}} \int d^{d+1} x \sqrt{-g}\left(R[g]-\frac{1}{2} \Gamma^{\mu} \Gamma_{\mu}\right),
$$

then reads, in harmonic gauge $\Gamma^{\mu} \equiv \Gamma_{\alpha \beta}^{\mu} g^{\alpha \beta}$,

$$
\begin{aligned}
S_{\mathrm{EH}}= & \int \mathrm{d}^{d+1} x \sqrt{-\gamma}\left\{\frac{1}{4}\left[(\nabla \sigma)^{2}-2\left(\nabla \sigma_{i j}\right)^{2}-\left(\dot{\sigma}^{2}-2\left(\dot{\sigma}_{i j}\right)^{2}\right) \mathrm{e}^{\frac{-c_{d} \phi}{\Lambda}}\right]-c_{d}\left[(\nabla \phi)^{2}-\dot{\phi}^{2} \mathrm{e}^{-\frac{c_{d} \phi}{\Lambda}}\right]\right. \\
& +\left[\frac{1}{2} F_{i j} F^{i j}+(\nabla \cdot \boldsymbol{A})^{2}-\dot{\boldsymbol{A}}^{2} \mathrm{e}^{-\frac{c_{d} \phi}{\Lambda}}\right] \mathrm{e}^{\frac{c_{d} \phi}{\Lambda}}+\frac{2}{\Lambda}\left[\left(F_{i j} \boldsymbol{A}^{i} \dot{\boldsymbol{A}}^{j}+\boldsymbol{A} \cdot \dot{\boldsymbol{A}}(\nabla \cdot \boldsymbol{A})\right) \mathrm{e}^{\frac{c_{d} \phi}{\Lambda}}-c_{d} \dot{\boldsymbol{\phi}} \boldsymbol{A} \cdot \nabla \boldsymbol{\phi}\right] \\
& +2 c_{d}(\dot{\boldsymbol{\phi}} \nabla \cdot \boldsymbol{A}-\dot{\boldsymbol{A}} \cdot \nabla \phi)+\frac{\dot{\sigma}_{i j}}{\Lambda}\left(-\delta^{i j} A_{l} \hat{\Gamma}_{k k}^{l}+2 A_{k} \hat{\Gamma}_{i j}^{k}-2 A^{i} \hat{\Gamma}_{k k}^{j}\right)-c_{d} \frac{\dot{\boldsymbol{\phi}}^{2} \boldsymbol{A}^{2}}{\Lambda^{2}} \\
& \left.-\frac{1}{\Lambda}\left(\frac{\sigma}{2} \delta^{i j}-\sigma^{i j}\right)\left(\sigma_{i k}{ }^{l} \sigma_{j l}{ }^{k}-\sigma_{i k}{ }^{, k} \sigma_{j l}{ }^{l}+\sigma_{, i} \sigma_{j k}{ }^{k}-\sigma_{i k, j} \sigma^{k}\right)\right\}+\cdots,
\end{aligned}
$$

where we used $\Lambda \equiv 1 / \sqrt{32 \pi G_{d}}, \sigma_{i j} \equiv \gamma_{i j}-\delta_{i j}$, and $\sigma \equiv \sigma_{i i}$. The above expression, along with the expansion of $S_{\mathrm{pp}}\left[x_{a}^{\alpha}\left(\tau_{a}\right)\right]$ in Eq. (2.1), allows us to derive all of the Feynman rules. For instance, the propagators for each field take the form (in mixed direct Fourier space)

$$
\frac{1}{2} P^{a a} \delta_{a b}(2 \pi)^{d} \delta^{(d)}(\boldsymbol{p}+\boldsymbol{q}) \mathcal{P}\left(\boldsymbol{p}^{2}, t_{1}, t_{2}\right) \delta\left(t_{1}-t_{2}\right),
$$

where $P^{\phi \phi}=-\frac{1}{c_{d}}, P^{A_{i} A_{j}}=\delta_{i j}, P^{\sigma_{i j} \sigma_{k l}}=-\left(\delta_{i k} \delta_{j l}+\delta_{i l} \delta_{j k}+\left(2-c_{d}\right) \delta_{i j} \delta_{k l}\right)$, and

$$
\mathcal{P}\left(p^{2}, t_{1}, t_{2}\right)=\frac{i}{\boldsymbol{p}^{2}-\partial_{t_{1}} \partial_{t_{2}}} .
$$

In the nonrelativistic limit the time derivatives are expanded in Taylor series, and ultimately applied to the external (worldline) sources. As discussed in Sec. VI, this is the reason for the spurious near-zone IR divergences.

All integrals required for the evaluation of the 4PN effective action can be reduced to combinations of the following master integrals (see Refs. [14,15] for details):

$$
\begin{gathered}
\int \frac{d^{d} p}{(2 \pi)^{d}} \frac{e^{i \boldsymbol{p} \cdot \boldsymbol{r}}}{\boldsymbol{p}^{2 a}}=2^{-2 a} \pi^{-d / 2} \frac{\Gamma(d / 2-a)}{\Gamma(a)} r^{2 a-d}, \\
\int \frac{d^{d} k}{(2 \pi)^{d}} \frac{1}{(\boldsymbol{k}-\boldsymbol{p})^{2 a} \boldsymbol{k}^{2 b}}=\frac{1}{(4 \pi)^{d / 2}} \frac{\Gamma(d / 2-a) \Gamma(d / 2-b) \Gamma(a+b-d / 2)}{\Gamma(a) \Gamma(b) \Gamma(d-a-b)},
\end{gathered}
$$

with the exception of some contributions at $\mathcal{O}\left(G^{5}\right)$, which can be mapped into four-loop (massless) two-point functions. The integrals involved in these diagrams have been computed in terms of more complex master integrals (see Ref. [18] for a detailed discussion, as well as Ref. [30]). Notice that Eq. (A5) can only have IR poles (for $a=\frac{d}{2}+n$, with integer $n \geq 0$ ), while Eq. (A6) may contain both IR and UV poles (e.g., the latter occurring when $a+b=\frac{d}{2}-n$ ).

\section{APPENDIX B: SPURIOUS IR/UV POLES}

The EFT for the radiation region in Eq. (2.6), augmented by the terms in Eq. (4.13), leads to several UV divergences in the conservative sector of the radiation-reaction force. After inserting the different (source) couplings into tail-type diagrams, similar to Fig. 3, we find

$$
\begin{aligned}
\mathcal{L}_{\phi^{3}, 4 \mathrm{PN}}^{\mathrm{UV}(\mathrm{far})}= & \frac{G^{2}}{\epsilon_{\mathrm{UV}}}\left[( M + T ^ { k k } ) \left(-\frac{1}{60}\left(2\left(I_{0}^{i j(3)}\right)^{2}+\left(I_{0}^{i i(3)}\right)^{2}\right)-\left(\dot{M}+T^{k k(1)}+\frac{1}{3} I_{0}^{k k(3)}\right)\left(\dot{M}+T^{i i(1)}\right)-\frac{1}{3}\left(\ddot{\boldsymbol{X}}^{i}+\ddot{\boldsymbol{T}}^{i}\right)^{2}\right.\right. \\
& \left.\left.-\frac{1}{15} I_{0}^{i k k(4)}\left(\ddot{\boldsymbol{X}}^{i}+\ddot{\boldsymbol{T}}^{i}\right)\right)-\frac{2}{3}\left(\dot{M}+T^{k k(1)}\right)\left(\ddot{\boldsymbol{X}}^{i}+\ddot{\boldsymbol{T}}^{i}\right)\left(\dot{\boldsymbol{X}}^{i}+\dot{\boldsymbol{T}}^{i}\right)+\frac{1}{30}\left(I_{0}^{k k(2)} \delta^{i j}+2 I_{0}^{i j(2)}\right)\left(\ddot{\boldsymbol{X}}^{i} \ddot{\boldsymbol{X}}^{j}+2 \dot{\boldsymbol{X}}^{i} \dddot{\boldsymbol{X}}^{j}\right)\right],
\end{aligned}
$$




$$
\begin{gathered}
\mathcal{L}_{A^{2} \phi, 4 \mathrm{PN}}^{\mathrm{UV}(\mathrm{far})}=\frac{G^{2}}{\epsilon_{\mathrm{UV}}}\left[\left(M+T^{k k}\right)\left(4 \dot{\boldsymbol{P}}^{2}+\frac{4}{3}\left(M^{i j(2)}\right)^{2}+\frac{4}{3} \dot{\boldsymbol{P}}^{i} M^{i k k(3)}\right)+\frac{2}{3} \dot{\boldsymbol{P}}^{2} I_{0}^{k k(2)}+\frac{8}{3} M^{i j(1)} \dot{\boldsymbol{P}}^{i} \dot{\boldsymbol{X}}^{j}\right], \\
\mathcal{L}_{\sigma^{2} \phi 4 \mathrm{PN}}^{\mathrm{UV}(\mathrm{far})}=\frac{2 G^{2} M}{\epsilon_{\mathrm{UV}}}\left[\left(T^{k k(1)}\right)^{2}-\left(T^{i j(1)}\right)^{2}\right],
\end{gathered}
$$

$$
\begin{gathered}
\mathcal{L}_{A \phi^{2}, 4 \mathrm{PN}}^{\mathrm{UV}(\mathrm{far})}=\frac{G^{2}}{3 \epsilon_{\mathrm{UV}}}\left[4 \ddot{\boldsymbol{X}}^{i} \boldsymbol{P}^{i}\left(\dot{M}+T^{k k(1)}\right)-\frac{2}{5} M^{k k(1)} \dot{\boldsymbol{X}}^{i} \dddot{\boldsymbol{X}}^{i}\right. \\
-\frac{1}{5} I_{0}^{k k(2)}\left(\dot{\boldsymbol{P}}^{i} \ddot{\boldsymbol{X}}^{i}+\boldsymbol{P}^{i} \dddot{\boldsymbol{X}}^{i}\right)-\frac{2}{5} \dot{M}_{i j}\left(\dot{\boldsymbol{X}}^{i} \dddot{\boldsymbol{X}}^{j}+\dot{\boldsymbol{X}}^{j} \dddot{\boldsymbol{X}}^{i}\right) \\
\left.-\frac{2}{5} I_{0}^{i j(2)}\left(\dot{\boldsymbol{P}}^{i} \ddot{\boldsymbol{X}}^{j}+\boldsymbol{P}^{i} \dddot{\boldsymbol{X}}^{j}\right)\right], \\
\mathcal{L}_{A^{2} \sigma, 4 \mathrm{PN}}^{\mathrm{UV}(\mathrm{far})}=-\frac{8 G^{2}}{3 \epsilon_{\mathrm{UV}}} T^{k k} \dot{\boldsymbol{P}}^{i} \dot{\boldsymbol{P}}^{i}, \\
\mathcal{L}_{\sigma \phi^{2}, 4 \mathrm{PN}}^{\mathrm{UV}(\mathrm{far})}=\frac{2 G^{2}}{15 \epsilon_{\mathrm{UV}}}\left(-T^{i j} \ddot{\boldsymbol{X}}^{i} \ddot{\boldsymbol{X}}^{j}+2 T^{k k} \ddot{\boldsymbol{X}}^{i} \ddot{\boldsymbol{X}}^{i}\right), \\
\mathcal{L}_{\phi A \sigma, 4 \mathrm{PN}}^{\mathrm{UV}(\mathrm{far})}=0,
\end{gathered}
$$

$$
\begin{aligned}
& \mathcal{L}_{A \sigma^{2}, 4 \mathrm{PN}}^{\mathrm{UV}(\mathrm{far})}=0, \\
& \mathcal{L}_{\sigma^{3}, 4 \mathrm{PN}}^{\mathrm{UV}(\mathrm{far})}=0,
\end{aligned}
$$

where we have also split the answer in terms of scalar, vector, and tensor modes, and reported the results in standard variables such that the variation of the action acts as usual. For instance, the $2 \mathrm{PN}$ and $3 \mathrm{PN}$ contributions proportional to $\dot{M}^{2}$ and $\ddot{X}^{2}$ mentioned in the text arise from the first two equations. Moreover, the $\phi^{3}, \sigma^{2} \phi$, and $A^{2} \phi$ couplings account for the contribution from the quadrupole moment leading to Eq. (2.10) at 4PN order. However, other (yet unphysical) terms appear, involving the following moments of the pseudo-stress-energy tensor, $\mathcal{T}^{\alpha \beta}$, expanded to the desired order:

$$
\begin{gathered}
M \equiv \int d^{3} \boldsymbol{x} \mathcal{T}^{00}=-\frac{G m_{1} m_{2}}{r}\left(1-\epsilon_{\mathrm{UV}} \log \bar{\mu} r\right)+\sum_{a} m_{a}\left(1+\frac{\boldsymbol{v}_{a}^{2}}{2}\right)+\cdots \\
\boldsymbol{X}^{i} \equiv \int d^{3} \boldsymbol{x} \mathcal{T}^{00} \boldsymbol{x}^{i}=\sum_{a \neq b} m_{a}\left[1+\frac{\boldsymbol{v}_{a}^{2}}{2}-\frac{G m_{b}}{2 r}\left(1-\epsilon_{\mathrm{UV}} \log \bar{\mu} r\right)\right] \boldsymbol{x}_{a}^{i}+\cdots \\
\boldsymbol{P}^{i} \equiv \int d^{3} \boldsymbol{x} \mathcal{T}^{0 i}=\sum_{a \neq b} m_{a}\left[\left(1+\frac{\boldsymbol{v}_{a}^{2}}{2}\right) \boldsymbol{v}_{a}^{i}-\frac{G m_{b}}{2 r}\left(\boldsymbol{v}_{a}^{i}+\frac{1}{r^{2}} \boldsymbol{r} \cdot \boldsymbol{v}_{a} \boldsymbol{r}^{i}\right)\left(1-\epsilon_{\mathrm{UV}} \log \bar{\mu} r\right)\right]+\cdots, \\
I_{0}^{i j} \equiv \int d^{3} \boldsymbol{x} \mathcal{T}^{00} \boldsymbol{x}^{i} \boldsymbol{x}^{j}=\sum_{a} m_{a} \boldsymbol{x}_{a}^{i} \boldsymbol{x}_{a}^{j}+\cdots \\
I_{0}^{i j k} \equiv \int d^{3} \boldsymbol{x} \mathcal{T}^{00} \boldsymbol{x}^{i} \boldsymbol{x}^{j} \boldsymbol{x}^{k}=\sum_{a} m_{a} \boldsymbol{x}_{a}^{i} \boldsymbol{x}_{a}^{j} \boldsymbol{x}_{a}^{k}+\cdots \\
M^{i j k} \equiv \int d^{3} \boldsymbol{x} \mathcal{T}^{0 i} \boldsymbol{x}^{j}=\sum_{a} m_{a} \boldsymbol{v}_{a}^{i} \boldsymbol{x}_{a}^{j}+\cdots, \\
T^{i j} \equiv \mathcal{T}^{0 i} \boldsymbol{x}^{j} \boldsymbol{x}^{k}=\sum_{a} m_{a} \boldsymbol{v}_{a}^{i} \boldsymbol{x}_{a}^{j} \boldsymbol{x}_{a}^{k}+\cdots \\
d^{3} \boldsymbol{x} \mathcal{T}^{i j}=\sum_{a} m_{a} \boldsymbol{v}_{a}^{i} \boldsymbol{v}_{a}^{j}-\frac{G m_{1} m_{2}}{r^{3}}\left(1-\epsilon_{\mathrm{UV}} \log \bar{\mu} r\right) \boldsymbol{r}^{i} \boldsymbol{r}^{j}+\cdots \\
T^{k j k} \equiv \int d^{3} \boldsymbol{x} \mathcal{T}^{i j} \boldsymbol{x}^{k}=\sum_{a \neq b} m_{a}\left[\boldsymbol{v}_{a}^{i} \boldsymbol{v}_{a}^{j}-\frac{G m_{b}}{2 r^{3}} \boldsymbol{r}^{i} \boldsymbol{r}^{j}\left(1-\epsilon_{\mathrm{UV}} \log \bar{\mu} r\right)\right] \boldsymbol{x}_{a}^{k} \\
\sum_{a \neq b} m_{a}\left[\boldsymbol{v}_{a}^{2}-\frac{G m_{b}}{2 r}\left(1-\epsilon_{\mathrm{UV}} \log \bar{\mu} r\right)\right] \boldsymbol{x}_{a}^{k}+\cdots
\end{gathered}
$$




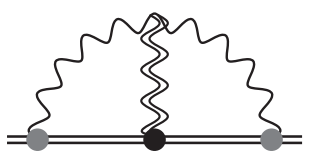

FIG. 6. Diagram depicting a contribution due to the tail effect to the conservative sector of the radiation-reaction force coming from the $A^{2} \sigma$ coupling. Following Ref. [39], we use a single wavy line to represent the vector mode $\boldsymbol{A}$, while the double wavy line accounts for the tensor $\sigma$. The total binary's mass-energy and moments of the pseudo-stress-energy tensor are described by the black and grey blobs, respectively. All permutations of the fields must be considered.

The second equality is obtained by matching the stress tensor to the two-body effective action, including both potential and radiation modes, after the former are integrated out; see, e.g., Refs. [12,43]. Notice that we have kept their values in $d$ dimensions, and also included the logarithmic terms. As we emphasized, even though the extra terms vanish on shell, they are essential for removing all of the unphysical poles in the intermediate computations. Combining all of these terms, we arrive at the expressions given in Eqs. (4.16)-(4.18).

It is instructive, however, to notice that the cancellation also occurs "polarization by polarization," or in other words, when the tensorial structure of the diagram is also taken into account. For example, to consider a specific case, let us look at the $A^{2} \sigma$ coupling. In the far zone, the relevant diagram(s) are summarized in Fig. 6. The result is given in Eq. (B2). Let us concentrate on the divergent parts, where we find

$$
\begin{aligned}
\mathcal{L}_{A^{2} \sigma 4 \mathrm{PN}}^{\mathrm{UV}(\mathrm{far})}= & -\frac{8}{3 \epsilon_{\mathrm{UV}}} G^{2} m_{1}^{2}\left[m_{2}\left(2 \boldsymbol{v}_{1}^{2}\left(\boldsymbol{a}_{1} \cdot \boldsymbol{a}_{2}\right)+\boldsymbol{a}_{1}^{2} \boldsymbol{v}_{2}^{2}\right)+m_{1} \boldsymbol{a}_{1}^{2} \boldsymbol{v}_{1}^{2}\right] \\
& +\frac{8}{3 \epsilon_{\mathrm{UV}}} \frac{G^{3} m_{1}^{2} m_{2}}{r}\left[m_{1} \boldsymbol{a}_{1}^{2}+m_{2} \boldsymbol{a}_{1} \cdot \boldsymbol{a}_{2}\right]+(1 \leftrightarrow 2) .
\end{aligned}
$$

Notice that it receives contributions both at $\mathcal{O}\left(G^{2}\right)$ and $\mathcal{O}\left(G^{3}\right)$; see Eqs. (B13) and (B18).

On the other hand, the diagrams associated with the near-zone computation are shown in Figs. 7 and 8 for the $G^{2}$ and $G^{3}$ corrections, respectively (plus mirror images). The result for the $G^{2}$ topologies to 4PN order (which involve an expansion in $p_{0} /|\boldsymbol{p}|$ for the would-be radiation modes) has both IR and UV divergences, given by

$$
\mathcal{L}_{\text {Fig. } 7}^{\mathrm{IR} / \mathrm{UV}}=\frac{8}{3} \frac{G^{2} m_{1}^{2} m_{2}}{\epsilon_{\mathrm{IR}}}\left[2 \boldsymbol{v}_{1}^{2}\left(\boldsymbol{a}_{1} \cdot \boldsymbol{a}_{2}\right)+\boldsymbol{a}_{1}^{2} \boldsymbol{v}_{2}^{2}\right]+\frac{8}{3} G^{2} m_{1}^{3}\left(\frac{1}{\epsilon_{\mathrm{IR}}}-\frac{1}{\epsilon_{\mathrm{UV}}}\right) \boldsymbol{a}_{1}^{2} \boldsymbol{v}_{1}^{2}
$$

for the sum of the first two diagrams plus the selfenergy contribution, respectively. Recall that at $\mathcal{O}\left(G^{2}\right)$ only IR divergences are present, except for the scaleless integral. The UV pole from the latter, shown in Eq. (B22), must be removed by the counterterms (see Sec. III B), whereas after the zero-bin subtraction the IR poles turn into UV divergences and exactly cancel the $G^{2}$ contribution in Eq. (B21), after adding the mirror diagrams.

The cancellation for topologies at $\mathcal{O}\left(G^{3}\right)$ is a bit more subtle at 4PN order. The divergent parts of the relevant diagrams are given by

$$
\begin{aligned}
\mathcal{L}_{\mathrm{Fig} .8(1 \mathrm{Ist})}^{\mathrm{IR} / \mathrm{VV}}= & \frac{G^{3} m_{1}^{3} m_{2}}{\epsilon_{\mathrm{UV}} r^{3}}\left[r\left(-4 \boldsymbol{v}_{1}^{2}\left(\boldsymbol{a}_{1} \cdot \boldsymbol{n}\right)-2\left(\boldsymbol{a}_{2} \cdot \boldsymbol{n}\right)\left(\boldsymbol{v}_{1} \cdot \boldsymbol{n}\right)^{2}+\frac{4}{3}\left(\boldsymbol{a}_{2} \cdot \boldsymbol{v}_{1}\right)\left(\boldsymbol{v}_{1} \cdot \boldsymbol{n}\right)\right)\right. \\
& -10\left(\boldsymbol{v}_{1} \cdot \boldsymbol{n}\right)^{2}\left(\boldsymbol{v}_{2} \cdot \boldsymbol{n}\right)^{2}+8 \boldsymbol{v}_{2}^{2}\left(\boldsymbol{v}_{1} \cdot \boldsymbol{n}\right)^{2}-14 \boldsymbol{v}_{1}^{2}\left(\boldsymbol{v}_{1} \cdot \boldsymbol{n}\right)\left(\boldsymbol{v}_{2} \cdot \boldsymbol{n}\right) \\
& +8\left(\boldsymbol{v}_{1} \cdot \boldsymbol{n}\right)\left(\boldsymbol{v}_{2} \cdot \boldsymbol{n}\right)\left(\boldsymbol{v}_{1} \cdot \boldsymbol{v}_{2}\right)+\frac{136}{5} \boldsymbol{v}_{1}^{2}\left(\boldsymbol{v}_{1} \cdot \boldsymbol{n}\right)^{2}-2 \boldsymbol{v}_{1}^{2} \boldsymbol{v}_{2}^{2}-\frac{4}{3}\left(\boldsymbol{v}_{1} \cdot \boldsymbol{v}_{2}\right)^{2} \\
& \left.+\frac{14}{3} \boldsymbol{v}_{1}^{2} \boldsymbol{v}_{1} \cdot \boldsymbol{v}_{2}-\frac{136}{15} \boldsymbol{v}_{1}^{4}\right]-\frac{8}{3 \epsilon_{\mathrm{IR}}} \frac{G^{3} m_{1}^{3} m_{2}}{r} \boldsymbol{a}_{1} \cdot \boldsymbol{a}_{1}, \\
\mathcal{L}_{\mathrm{Fig} .8 \text { (2nd) }}^{\mathrm{IR} / \mathrm{UV}} & -\frac{8}{3 \epsilon_{\mathrm{IR}}} \frac{G^{3} m_{1}^{2} m_{2}^{2}}{r} \boldsymbol{a}_{1} \cdot \boldsymbol{a}_{2}
\end{aligned}
$$



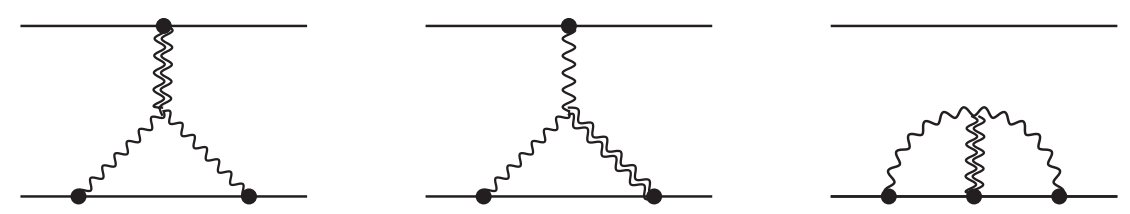

FIG. 7. Near-zone Feynman diagram at $\mathcal{O}\left(G^{2}\right)$ involving vector (single wavy) and tensor (double wavy) modes, associated with the far-zone conservative contributions in Fig. 6, at the same order. Mirror images must be added.
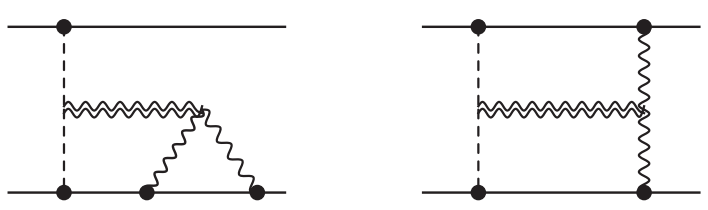

FIG. 8. Near-zone Feynman diagram at $\mathcal{O}\left(G^{3}\right)$ associated with the far-zone conservative contributions in Fig. 6, at the same order. The dashed line represents the scalar mode. Mirror images must be added.

for the first and second diagrams, respectively. Notice that, while the latter is IR divergent at 4PN order, the former has both IR and UV poles. A diagram similar to the first graph in Fig. 8, with the $A$ and $\phi$ fields exchanged (not shown), is only UV divergent. For the other topologies at $G^{3}$, the first diagram shown in Fig. 1 does not contribute for the given modes involved, and neither does the second self-energy diagram in Fig. 4. (Moreover, the first graph in Fig. 1 is always UV divergent for every nonvanishing polarization at 4PN order.) There are no other contributions to $4 \mathrm{PN}$ order.
Once again, all of the UV poles are removed by counterterms, while the IR singularity is handled by the zero-bin subtraction. As expected, the leftover UV pole cancels out against the associated contribution in Eq. (B21) at $\mathcal{O}\left(G^{3}\right)$.

Notice that in the graphs at this order, the $\sigma$ propagator is attached to the scalar potential mode rather than directly to the worldline. This is expected, since the radiation field also couples to the binding potential. In the far zone, this coupling is encoded in the expansion of the multipole moments in powers of Newton's constant. In our case, it is implicit in the $\mathcal{O}(G)$ corrections to the moment of the pseudo-stress-energy tensor in Eq. (B18). (Something similar occurs when we match the multipole moments to compute the radiated power; see, e.g., Refs. [43-45].) We can show that, for the reasons discussed in Sec. VI, a similar cancellation of spurious divergences is at work for each one of the polarizations involved, order by order in $G$, to $4 \mathrm{PN}$ order. ${ }^{32}$

\footnotetext{
${ }^{32}$ It is straightforward to show, for instance, that there is no contribution from the $A \sigma^{2}, \phi A \sigma$, and $\sigma^{3}$ couplings at 4PN order from either side, i.e., Eqs. (B8)-(B10).
}

[1] B. Abbott et al. (LIGO and Virgo Collaborations), Observation of Gravitational Waves from a Binary Black Hole Merger, Phys. Rev. Lett. 116, 061102 (2016).

[2] A. Buonanno and B. S. Sathyaprakash, Review in General Relativity and Gravitation: A Centennial Perspective, edited by A. Ashtekar, B. K. Berger, J. Isenberg, and M. A. H. MacCallum [arXiv:1410.7832].

[3] R. A. Porto, The tune of love and the nature(ness) of spacetime, Fortschr. Phys. 64, 723 (2016).

[4] R. A. Porto, The music of the spheres: The dawn of gravitational wave science, arXiv:1703.06440.

[5] B. P. Abbott et al. (LIGO Scientific and Virgo Collaborations), GWTC-1: A gravitational-wave transient catalog of compact binary mergers observed by LIGO and Virgo during the first and second observing runs, arXiv: 1811.12907.

[6] L. Blanchet, Gravitational radiation from post-Newtonian sources and inspiralling compact binaries, Living Rev. Relativity 17, 2 (2014).

[7] G. Schäfer and P. Jaranowski, Hamiltonian formulation of general relativity and post-Newtonian dynamics of compact binaries, Living Rev. Relativity 21, 7 (2018).
[8] W. Goldberger and I. Rothstein, An effective field theory of gravity for extended objects, Phys. Rev. D 73, 104029 (2006).

[9] S. Foffa and R. Sturani, Effective field theory methods to model compact binaries, Classical Quantum Gravity 31, 043001 (2014).

[10] V. Cardoso and R. A. Porto, Analytic approximations, perturbation theory, effective field theory methods and their applications, Gen. Relativ. Gravit. 46, 1682 (2014).

[11] I. Rothstein, Progress in effective field theory approach to the binary inspiral problem, Gen. Relativ. Gravit. 46, 1726 (2014).

[12] R. A. Porto, The effective field theorist's approach to gravitational dynamics, Phys. Rep. 633, 1 (2016).

[13] M. Levi, Effective field theories of post-Newtonian gravity: A comprehensive review, arXiv:1807.01699.

[14] S. Foffa and R. Sturani, preceding paper, Conservative dynamics of binary systems to fourth post-Newtonian order in the EFT Approach I: Regularized lagrangian, Phys. Rev. D, 100024047 (2019).

[15] S. Foffa and R. Sturani, Dynamics of the gravitational twobody problem at fourth post-Newtonian order and at 
quadratic order in the Newton constant, Phys. Rev. D 87, 064011 (2013).

[16] S. Foffa and R. Sturani, Tail terms in gravitational radiation reaction via effective field theory, Phys. Rev. D 87, 044056 (2013).

[17] C. Galley, A. Leibovich, R. A. Porto, and A. Ross, Tail effect in gravitational radiation reaction: Time nonlocality and renormalization group evolution, Phys. Rev. D 93, 124010 (2016).

[18] S. Foffa, P. Mastrolia, R. Sturani, and C. Sturm, Effective field theory approach to the gravitational two-body dynamics, at fourth post-Newtonian order and quintic in the Newton constant, Phys. Rev. D 95, 104009 (2017).

[19] R. A. Porto, Lamb shift and the gravitational binding energy for binary black holes, Phys. Rev. D 96, 024063 (2017).

[20] R. A. Porto and I. Rothstein, Apparent ambiguities in the post-Newtonian expansion for binary systems, Phys. Rev. D 96, 024062 (2017).

[21] P. Jaranowski and G. Schäfer, Dimensional regularization of local singularities in the 4th post-Newtonian two-point-mass Hamiltonian, Phys. Rev. D 87, 081503 (2013).

[22] D. Bini and T. Damour, Analytical determination of the two-body gravitational interaction potential at the fourth post-Newtonian approximation, Phys. Rev. D 87, 121501 (2013).

[23] T. Damour, P. Jaranowski, and G. Schäfer, Nonlocal-in-time action for the fourth post-Newtonian conservative dynamics of two-body systems, Phys. Rev. D 89, 064058 (2014).

[24] P. Jaranowski and G. Schäfer, Derivation of local-in-time fourth post-Newtonian ADM Hamiltonian for spinless compact binaries, Phys. Rev. D 92, 124043 (2015).

[25] T. Damour, P. Jaranowski, and G. Schäfer, Conservative dynamics of two-body systems at the fourth post-Newtonian approximation of general relativity, Phys. Rev. D 93, 084014 (2016).

[26] L. Bernard, L. Blanchet, A. Bohé, G. Faye, and S. Marsat, Fokker action of nonspinning compact binaries at the fourth post-Newtonian approximation, Phys. Rev. D 93, 084037 (2016).

[27] L. Bernard, L. Blanchet, A. Bohé, G. Faye, and S. Marsat, Energy and periastron advance of compact binaries on circular orbits at the fourth post-Newtonian order, Phys. Rev. D 95, 044026 (2017).

[28] L. Bernard, L. Blanchet, A. Bohé, G. Faye, and S. Marsat, Dimensional regularization of the IR divergences in the Fokker action of point-particle binaries at the fourth postNewtonian order, Phys. Rev. D 96, 104043 (2017).

[29] T. Marchand, L. Bernard, L. Blanchet, and G. Faye, Ambiguity-free completion of the equations of motion of compact binary systems at the fourth post-Newtonian order, Phys. Rev. D 97, 044023 (2018).

[30] T. Damour and P. Jaranowski, Four-loop static contribution to the gravitational interaction potential of two point masses, Phys. Rev. D 95, 084005 (2017).

[31] S. Foffa, P. Mastrolia, R. Sturani, C. Sturm, and W. J. Torres Bobadilla, Static two-body potential at fifth post-Newtonian order, arXiv:1902.10571.

[32] J. Blümlein, A. Maier, and P. Marquard, Five-loop static contribution to the gravitational interaction potential of two point masses, arXiv:1902.11180.
[33] A. V. Manohar and I. W. Stewart, The zero-bin and mode factorization in quantum field theory, Phys. Rev. D 76, 074002 (2007).

[34] W. Goldberger and I. Rothstein, Dissipative effects in the Worldline approach to black hole dynamics, Phys. Rev. D 73, 104030 (2006).

[35] R. A. Porto, Post-Newtonian corrections to the motion of spinning bodies in NRGR, Phys. Rev. D 73, 104031 (2006).

[36] R. A. Porto and I. Rothstein, The Hyperfine Einstein-InfeldHoffmann Potential, Phys. Rev. Lett. 97, 021101 (2006).

[37] R. A. Porto, Absorption effects due to spin in the Worldline approach to black hole dynamics, Phys. Rev. D 77, 064026 (2008).

[38] R. A. Porto and I. Z. Rothstein, Comment on on the next-toleading order gravitational spin(1)—spin(2) dynamics, edited by J. Steinhoff et al., arXiv:0712.2032.

[39] J. B. Gilmore and A. Ross, Effective field theory calculation of second post-Newtonian binary dynamics, Phys. Rev. D 78, 124021 (2008).

[40] R. A. Porto and I. Rothstein, Spin(1)Spin(2) effects in the motion of inspiralling compact binaries at third order in the post-Newtonian expansion, Phys. Rev. D 78, 044012 (2008); Erratum, Phys. Rev. D 81, 029904(E) (2010).

[41] R. A. Porto and I. Rothstein, Next-to-leading order spin(1)spin(1) effects in the motion of inspiralling compact binaries, Phys. Rev. D 78, 044013 (2008); Erratum, Phys. Rev. D 81, 029905(E) (2010).

[42] R. A. Porto, Next-to-leading order spin-orbit effects in the motion of inspiralling compact binaries, Classical Quantum Gravity 27, 205001 (2010).

[43] W. Goldberger and A. Ross, Gravitational radiative corrections from effective field theory, Phys. Rev. D 81, 124015 (2010).

[44] R. A. Porto, A. Ross, and I. Rothstein, Spin-induced multipole moments for the gravitational wave flux from binary inspirals to third post-Newtonian order, J. Cosmol. Astropart. Phys. 03 (2011) 009.

[45] R. A. Porto, A. Ross, and I. Rothstein, Spin-induced multipole moments for the gravitational wave amplitude from binary inspirals to 2.5 post-Newtonian order, J. Cosmol. Astropart. Phys. 09 (2012) 028.

[46] S. Foffa and R. Sturani, Effective field theory calculation of conservative binary dynamics at third post-Newtonian order, Phys. Rev. D 84, 044031 (2011).

[47] A. Ross, Multipole Expansion at the level of the action, Phys. Rev. D 85, 125033 (2012).

[48] W. D. Goldberger, A. Ross, and I. Z. Rothstein, Black hole mass dynamics and renormalization group evolution, Phys. Rev. D 89, 124033 (2014).

[49] M. Levi and J. Steinhoff, Complete conservative dynamics for inspiralling compact binaries with spins at fourth postNewtonian order, arXiv:1607.04252.

[50] W. Goldberger, Les Houches lectures on effective field theories and gravitational radiation, in Les Houches Summer School-Session 86: Particle Physics and Cosmology (Elsevier, New York, 2007).

[51] R. A. Porto and R. Sturani, Post-Newtonian corrections via an effective field theory approach, in Les Houches Summer School-Session 86: Particle Physics and Cosmology (Elsevier, New York, 2007). 
[52] C. R. Galley, A. K. Leibovich, and I. Z. Rothstein, Finite Size Corrections to the Radiation Reaction Force in Classical Electrodynamics, Phys. Rev. Lett. 105, 094802 (2010).

[53] P. Forgacs, T. Herpay, and P. Kovacs, Comment on Finite Size Corrections to the Radiation Reaction Force in Classical Electrodynamics, Phys. Rev. Lett. 109, 029501 (2012).

[54] C. R. Galley, A. K. Leibovich, and I. Z. Rothstein, Reply to Comment on Finite Size Corrections to the Radiation Reaction Force in Classical Electrodynamics, Phys. Rev. Lett. 109, 029502 (2012).

[55] T. Damour and A. Nagar, Relativistic tidal properties of neutron stars, Phys. Rev. D 80, 084035 (2009).

[56] T. Binnington and E. Poisson, Relativistic theory of tidal love numbers, Phys. Rev. D 80, 084018 (2009).

[57] B. Kol and M. Smolkin, Black hole stereotyping: Induced gravito-static polarization, J. High Energy Phys. 02 (2012) 010.

[58] C. R. Galley, Classical Mechanics of Nonconservative Systems, Phys. Rev. Lett. 110, 174301 (2013).

[59] C. R. Galley, D. Tsang, and L. C. Stein, The principle of stationary nonconservative action for classical mechanics and field theories, arXiv:1412.3082.

[60] L. Blanchet, T. Damour, and G. Esposito-Farese, Dimensional regularization of the third postNewtonian dynamics of point particles in harmonic coordinates, Phys. Rev. D 69 , 124007 (2004).

[61] L. Blanchet and T. Damour, Tail transported temporal correlations in the dynamics of a gravitating system, Phys. Rev. D 37, 1410 (1988).

[62] L. Blanchet and G. Faye, Lorentzian regularization and the problem of point-like particles in general relativity, J. Math. Phys. (N.Y.) 42, 4391 (2001).

[63] M. Beneke and V. A. Smirnov, Asymptotic expansion of Feynman integrals near threshold, Nucl. Phys. B522, 321 (1998).

[64] I.Z. Rothstein, TASI lectures on effective field theories, arXiv:hep-ph/0308266.

[65] A. V. Manohar, Introduction to effective field theories, in Les Houches summer school: EFT in Particle Physics and Cosmology Les Houches, Chamonix Valley, France, 2017 (2018), https://indico.in2p3.fr/event/13465/.

[66] D. Neill and I. Z. Rothstein, Classical space-times from the S matrix, Nucl. Phys. B877, 177 (2013).
[67] C. Cheung, I. Z. Rothstein, and M. P. Solon, From Scattering Amplitudes to Classical Potentials in the PostMinkowskian Expansion, Phys. Rev. Lett. 121, 251101 (2018).

[68] Z. Bern, C. Cheung, R. Roiban, C.-H. Shen, M. P. Solon, and M. Zeng, Scattering Amplitudes and the Conservative Hamiltonian for Binary Systems at Third Post-Minkowskian Order, Phys. Rev. Lett. 122, 201603 (2019).

[69] B. R. Holstein and A. Ross, Spin effects in long range gravitational scattering, arXiv:0802.0716.

[70] C. Galley and R. A. Porto, Gravitational self-force in the ultra-relativistic limit: The large- $N$ expansion, J. High Energy Phys. 11 (2013) 096.

[71] V. Vaidya, Gravitational spin Hamiltonians from the $\mathrm{S}$ matrix, Phys. Rev. D 91, 024017 (2015).

[72] T. Damour, High-energy gravitational scattering and the general relativistic two-body problem, Phys. Rev. D 97, 044038 (2018).

[73] A. Guevara, A. Ochirov, and J. Vines, Scattering of spinning black holes from exponentiated soft factors, arXiv: 1812.06895 .

[74] A. Laddha and A. Sen, Observational signature of the logarithmic terms in the soft graviton theorem, arXiv: 1806.01872 .

[75] D. A. Kosower, B. Maybee, and D. O'Connell, Amplitudes, observables, and classical scattering, J. High Energy Phys. 02 (2019) 137.

[76] S. Caron-Huot and Z. Zahraee, Integrability of black hole orbits in maximal supergravity, arXiv:1810.04694.

[77] J. Vines, J. Steinhoff, and A. Buonanno, Spinning-blackhole scattering and the test-black-hole limit at second postMinkowskian order, Phys. Rev. D 99, 064054 (2019).

[78] A. Addazi, M. Bianchi, and G. Veneziano, Soft gravitational radiation from ultra-relativistic collisions at sub- and subsub-leading order, J. High Energy Phys. 05 (2019) 050.

[79] A. Antonelli, A. Buonanno, J. Steinhoff, M. van de Meent, and J. Vines, Energetics of two-body Hamiltonians in postMinkowskian gravity, Phys. Rev. D 99, 104004 (2019).

[80] J. M. Henn and B. Mistlberger, Four-graviton scattering to three loops in $\mathcal{N}=8$ supergravity, J. High Energy Phys. 05 (2019) 023.

[81] T. Damour, Gravitational radiation and the motion of compact bodies, in Les Houches Summer School on Gravitational Radiation Les Houches, France, 1982 (1982), http://inspirehep.net/record/964974?ln=en. 